\title{
1 Atypical B cells are a normal component of immune responses to vaccination and infection in
}

2 humans

3

4 Henry J. Sutton ${ }^{1 *}$, Racheal Aye ${ }^{1,2^{*}}$, Azza H. Idris ${ }^{3}$, Rachel Vistein ${ }^{3}$, Eunice Nduati ${ }^{2,4}$, Oscar Kai²,

5 Jedida Mwacharo ${ }^{2}, \mathrm{Xi} \mathrm{Li}^{1}$, Xin $\mathrm{Gao}^{1}$, T. Daniel Andrews ${ }^{1}$, Marios Koutsakos ${ }^{5}$, Thi H. O. Nguyen ${ }^{5}$,

6 Maxim Nekrasov ${ }^{6}$, Peter Milburn ${ }^{6}$, Auda Ethala ${ }^{7}$, Andrea A. Berry ${ }^{8}$, Natasha $\mathrm{KC}^{9}$, Sumana

7 Chakravarty ${ }^{9}$, B. Kim Lee Sim $^{9}$, Adam K. Wheatley ${ }^{5,10}$, Stephen J. Kent ${ }^{5,10}$, Stephen L. Hoffman ${ }^{9}$,

8 Kirsten E. Lyke ${ }^{8}$, Philip Bejon ${ }^{2,4}$, Fabio Luciani ${ }^{7}$, Katherine Kedzierska ${ }^{5}$, Robert A. Seder ${ }^{3}$, Francis M.

$9 \quad \mathrm{Ndungu}^{2,4}$ and Ian A. Cockburn ${ }^{1 \dagger}$

11 1. Department of Immunology and Infectious Disease, John Curtin School of Medical Research, The

12 Australian National University

13 2. KEMRI - Wellcome Research Programme/Centre for Geographical Medicine Research

14 (Coast).

15 3. Vaccine Research Center, National Institutes of Allergy and Infectious Disease, National Institutes 16 of Health.

17 4. Centre for Tropical Medicine and Global Health, Nuffield Department of Medicine, University of 18 Oxford

19 5. Department of Microbiology and Immunology, Peter Doherty Institute, University of Melbourne

20 6. Australian Cancer Research Foundation Biomedical Resource Facility, John Curtin School of

21 Medical Research, The Australian National University

22 7. School of Medical Science, and Kirby Institute, University of New South Wales, Sydney Australia

23 8. Center for Vaccine Development and Global Health, University of Maryland School of Medicine,

24 Baltimore, MD 21201, USA 
bioRxiv preprint doi: https://doi.org/10.1101/2020.05.28.120808; this version posted May 28, 2020. The copyright holder for this preprint (which

was not certified by peer review) is the author/funder, who has granted bioRxiv a license to display the preprint in perpetuity. It is made available under aCC-BY-NC-ND 4.0 International license.

25 9. Sanaria Inc., Rockville, MD 20850, USA

10. ARC Centre of Excellence in Convergent Bio-Nano Science and Technology, The University of

Melbourne

$27 *$ Equal contribution

28

$29+$ Lead Contact: ian.cockburn@anu.edu.au; tel: ${ }^{+} 61261254619$

30

31 Keywords: Malaria; Influenza; Single Cell RNA-seq; B cell memory; Atypical B cells; Vaccination 


\section{Abstract}

34 The full diversity of the circulating human B cell compartment is unknown. Flow cytometry analysis

35 suggests that in addition to naïve and memory B cells, there exists a population of CD11c ${ }^{+}, \mathrm{CD}^{-7^{-}}$

36 CD21" "atypical" B cells, that are associated with chronic or recurrent infection and autoimmunity. We

37 used single cell RNA-seq approaches to examine the diversity of both antigen-specific B cells and total

38 B cells in healthy subjects and individuals naturally-exposed to recurrent malaria infections. This

39 analysis revealed two B cell lineages: a classical lineage of activated and resting memory B cells, and

40 an atypical-like lineage. Surprisingly, the atypical lineage was common in both malaria exposed

41 individuals and non-exposed healthy controls. Using barcoded antibodies in conjunction with our

42 transcriptomic data, we found that atypical lineage cells in healthy individuals lack many atypical B

43 markers and thus represent an undercounted cryptic population. We further determined using antigen

44 specific probes that atypical cells can be induced by primary vaccination in humans and can be recalled

45 upon boosting. Collectively these data suggest that atypical cells are not necessarily pathogenic but can

46 be a normal component of $\mathrm{B}$ responses to antigen. 


\section{Introduction}

49 The majority of currently approved vaccines require the generation of an effective antibody response to

50 provide long term immune protection (Plotkin, 2010). An effective antibody response requires the

51 formation of germinal centers (GCs) to produce somatically hypermutated and affinity matured long-

52 lived plasma cells (LLPCs) that secrete high-affinity antibody as well as antigen-experienced

53 "memory" B cells (MBCs) that are primed to produce a faster, larger and more effective response upon

54 secondary exposure (Tangye et al., 2003). In humans, circulating human B cells have been classified

55 based on the expression of the surface proteins CD38, CD27 and CD21. Plasma cells (PCs) express

56 high levels of CD38 and CD27 (Horst et al., 2002) while CD27 $7^{+}$D2 $1^{+} \mathrm{B}$ cells are considered to be

57 MBCs (Klein et al., 1997; Tangye et al., 1998). These CD27 cells show high levels of affinity

58 maturation and readily differentiate into antibody secreting PCs after stimulation compared to CD27-

$59 \mathrm{CD}_{21^{+}}$naïve cells (Good et al., 2009; Tangye et al., 2003). Populations of CD27 $7^{+}$CD21- B cells have

60 also been described, which have previously been associated with an activated B cell phenotype,

61 predisposed to differentiate into PCs (Avery et al., 2005; Lau et al., 2017). Conversely a subset of B

62 cells that are $\mathrm{CD} 27^{-} \mathrm{CD} 21^{-}$have also been observed, originally in tonsils and later in peripheral blood

63 (Ehrhardt et al., 2005; Fecteau et al., 2006). These cells, commonly referred to as atypical B cells

64 (atBC), are observed at high frequencies in conditions of chronic antigen stimulation such as infection

65 with HIV or malaria (Moir et al., 2008; Weiss et al., 2009) or autoimmune disease (Isnardi et al., 2010;

66 Wei et al., 2007).

67

68 Because they are often found in chronic infection and autoimmune disease, atBCs are usually

69 considered to be a population of anergic or exhausted B cells that arise due to chronic antigenic

70 stimulation. In support of this, atBCs typically express high levels of inhibitory receptors such as those 
71 belonging to the family of Fc-receptor-like (FCRL) molecules, as well as having muted BCR signaling

72 and display little to no capacity to differentiate into PCs following BCR stimulation in vitro (Moir et

73 al., 2008; Portugal et al., 2015; Sullivan et al., 2015). However, not all data indicate that atBCs are an

74 exhausted population. While SLE patients with high disease scores carry high numbers of atBCs, a

75 recent study suggested that these are short-lived activated cells, in the process of differentiating into

76 PCs (Jenks et al., 2018). Similarly, it has been shown that BCRs used by atBCs specific to Plasmodium

77 falciparum could also be found contributing to the anti-P. falciparum antibody response (Muellenbeck

78 et al., 2013). Furthermore, studies in mice show that short-lived, recently activated B cells express high

79 levels of CD11 $\mathrm{c}^{+}$and have similar gene expression patterns to human atBCs (Kim et al., 2019; Perez-

80 Mazliah et al., 2018).

81

82 To better understand the heterogeneity of the circulating B cell response in humans, and gain insight

83 into the role of atBCs, we performed single cell RNA-seq on antigen-specific B cells from malaria

84 exposed adults, we compared these data to single-cell RNA-seq on non-antigen specific B cells from

85 both malaria-exposed and non-exposed individuals. Finally, we examined the phenotypic profile when

86 antigen specific atBCs and MBCs arise in the response to vaccination. Collectively we found that even

87 non-exposed individuals carry high numbers of cells that express an atBC transcriptomic signature. Our

88 vaccination studies revealed that antigen-specific atBCs arise following a primary immune response

89 and are able to respond upon secondary exposure, suggesting that atBCs may have functional role in

90 the human humoral response. 
92

93

94

95

111 Following quality control steps, a total of 163 transcriptomes from the 11 individuals were obtained

\section{Results}

Single cell RNA-seq reveals three distinct populations of antigen-experienced circulating $B$ cells

The initial studies focused on the transcriptional diversity of the circulating B cell populations in malaria-vaccinated and -exposed humans by single-cell RNA sequencing (scRNA-seq). Specifically, we isolated $\mathrm{CD}_{19}, \mathrm{CD} 20^{+} \mathrm{B}$ cells that (i) were class switched i.e. $\mathrm{IgD}^{-}$and (ii) bound specific antigens. P. falciparum circumsporozoite (PfCSP) specific cells were isolated from the peripheral blood of five Kenyan children 6.5 and 74 months after receiving the last dose of the CSP-based RTS,S vaccine. To further examine the response to natural exposure to malaria PfCSP specific B cells, as well as B cells specific for the P. falciparum merozoite surface protein-1 (PfMSP1) were also sorted from 6 adult Kenyans from an area of moderate to high malaria transmission (Figure 1A); details of study subjects are given in Table S1). Individuals in this population carry high numbers of circulating atBCs as described using the absence of CD21 and CD27 as markers (Aye et al., 2020). We also sorted tetanus toxoid (TT) specific B cells from the adult subjects; which we have previously shown to have a more classical MBC phenotype (Aye et al., 2020). Because B cells specific for a given antigen are rare within an individual, only $\sim 10-50$ antigen specific cells could be sorted per sample. Accordingly, we used a modified version of the relatively low throughput Smart-seq2 protocol (Picelli et al., 2014) to obtain transcriptomes of the individual cells. and pooled for analysis using the R package, Seurat (Butler et al., 2018). Unsupervised hierarchical clustering grouped the cells into 3 clusters (Figure 1B-C). Differentially expressed genes (DEGs) were identified for each cluster using the Wilcox test to calculate the difference between the average 
115 expression by cells in the cluster against the average expression by all cells not in the cluster. DEGs

116 with an average log-fold change higher than 0.25 were used for further analysis. Gene set enrichment

117 analysis (GSEA) showed that cluster 1 had many DEGs associated with the atypical B cell (atBC)

118 phenotype (Portugal et al., 2015; Sullivan et al., 2015), such as FCRL5, FCRL3, ITGB2, ITGAX,

119 TNFRSF1B, LILRB1, CD19 and MS4A1 (Figure 1D; Figure S1A-B). Cluster 2 expressed the lymphoid

120 homing gene $C C R 7$ and the antiproliferative $B T G 1$ gene (Figure 1D; Figure S1B) suggesting that this

121 may be a quiescent resting/central B cell memory subset and therefore were classified as memory B

122 cells (MBC), while GSEA analysis revealed that these cells had a transcriptional profile similar to

123 naïve B cells (Figure 1D; Figure S1A). Cluster 3 expressed high levels of $C X C R 3$ and was found by

124 GSEA to be somewhat enriched for genes associated with previously described activated B cells

125 (ABCs) (Ellebedy et al., 2016) including high expression CSK and CD52, however a similar level of

$126 \mathrm{ABC}$ gene enrichment was also seen in the cluster 1 (Figure 1D; Figure S1A-B) therefore we did not

127 assign a designation to these cells.

128

129 Because cells were index sorted prior to sequencing, we could also measure the surface protein

130 expression on each cell, allowing us to investigate the expression of CD27 and CD21, the markers

131 traditionally used to distinguish different B cell types in humans. Strikingly, only $44.7 \%$ of cells in the

132 atBC cluster had the $\mathrm{CD} 27^{-} \mathrm{CD} 21^{-}$phenotype typically used to describe atBCs. Similarly, only $41.2 \%$

133 of cluster 3 cells had the $\mathrm{CD} 27^{+} \mathrm{CD} 21^{-}$phenotype of activated B cells. Finally, $37.5 \%$ of MBCs were

$134 \mathrm{CD}_{2} 7^{+} \mathrm{CD} 21^{+}$suggesting that there may be distinct transcriptional signatures that suggest greater

135 heterogeneity than using the canonical cell surface markers used to delineate memory B cell subsets

136 (Figure S1C-D).

137 
138 We have previously reported V(D)J sequences for the adult cells reconstructed using VDJpuzzle

139 software (Aye et al., 2020; Rizzetto et al., 2018) we further extended this analysis to cells from the

140 children analyzed in this study to V(D)J and isotype sequence from a total of 121/163 cells. This

141 analysis revealed that all populations, including MBCs had undergone somatic hypermutation (SHM),

142 though levels of SHM were slightly lower in MBCs, and - consistent with previous reports (Aye et al.,

143 2020; Murugan et al., 2018; Tan et al., 2018) - CSP-specific B cells (Figure 1E). This analysis further

144 revealed no strong association between antibody subclass and any population of memory cells (Figure

145 1F). In contrast when we subdivided the populations by antigen specificity we found that PfCSP-

146 specific B cells were predominantly atBCs which may be consistent with continuous exposure to low

147 levels of this antigen via repeated P falciparum infections (Figure 1G; Figure S1E). Surprisingly -

148 given the association of malaria exposure with atypical B cells - most PfMSP1 specific cells mapped to

149 cluster 3 rather than the atBC population; finally, Tetanus Toxoid specific cells were mostly MBCs

150 which is consistent with the absence of ongoing antigenic exposure (Figure 1G; Figure S1E). Overall

151 our data on antigen-specific cells enables us to identify 3 distinct populations of circulating B cells.

High throughput single-cell analysis identifies atBC, $M B C$ and ABC populations in both malaria-

exposed and non-exposed donors

156 We next wanted to know if the 3 subsets of circulating B cells we identified were specific to malaria or 157 reflected B cell memory in general. Moreover, we were concerned that the association of antigen with 158 cell populations, while striking, could be a result of cells of different specificities coming from 159 different donors and thus our analysis might be confounded by batch effects (Figure S1E-F). Finally, 160 we wanted to sample a larger number of cells as the relatively small number of cells analyzed may not 161 have allowed us to discern smaller populations of B cells. We therefore used the 10x Chromium 
162 platform to sequence single $\mathrm{CD} 20^{+} \mathrm{CD} 19^{+} \mathrm{IgD}^{-}$memory B cells, regardless of antigen specificity,

163 sorted from the PBMCs of two non-exposed donors (Non-Exp) and two malaria-exposed (Malaria-Exp)

164 donors (Table S1). We also included barcoded antibodies specific for CD11c, CXCR3, CD21 and

165 CD27 to perform Cellular Indexing of Transcriptomes and Epitopes by sequencing (CITE-seq) analysis

166 linking surface protein expression to transcriptomic data (Stoeckius et al., 2017). We chose these

167 markers based on our Smart-seq2 experiment and to reconcile our data with established markers.

168 Finally, we used single cell immune profiling to obtain paired heavy and light chain V(D)J chain 169 sequences for the BCR of each individual B cell.

171 After quality control steps, for the malaria-exposed donors, 1448 (Malaria-Exp 1) and 5719 (Malaria-

172 Exp 2) cells with median genes per cell of 1576 and 1652 respectively were sequenced. While in the 173 non-exposed individuals a further 2252 (Non-Exp 1) and 3561 (Non-Exp 2) cells were sequenced with 174 median genes per cell of 1668 and 1535 respectively. Unsupervised clustering using Seurat was 175 performed on each sample to identify any non-B cell clusters, to be removed before combining samples 176 together (Figure S2A). Strikingly, in one of the exposed individuals (Malaria-Exp 1), we identified a 177 cluster enriched for CD5 and BCL2 in which all cells expressed the same heavy and light chain 178 immunoglobulin genes (IGHV7-81 and IGKV1-8). We concluded that these cells might be from a 179 premalignant B cell clonal expansion and were subsequently removed from further analysis (Figure $180 \mathrm{~S} 2 \mathrm{~A})$.

181

182 Following removal of non-B cell populations, we used Seurat's integration feature to remove batch 183 effects between samples and combine all 4 into one integrated dataset (Figure 2A). Unsupervised 184 clustering was then performed on this combined dataset of 12621 cells, which revealed 11 conserved 185 clusters (Figure 2A; Figure S2B). Three of these clusters which were more distantly related to the 
186 others appeared to correspond to naïve B cells, PCs and a population of cells expressing high levels of

187 proliferation markers (Figure 2B; Figure S2B). The PC cluster could be discerned by the high

188 expression of the transcription factors $X B P 1, I R F 4$ and PRDM1 (Figure S3A), which are all associated

189 with controlling PC differentiation and maintenance (Klein et al., 2006; Reimold et al., 2001; Shaffer et

190 al., 2002). The naïve cluster was characterized by expression of $I G H D$, as well as $B A C H 2$ and $B T G 1$

191 (Figure S3A) which are transcriptional repressors associated with cellular quiescence (Guehenneux et

192 al., 1997; Muto et al., 1998; Tsukumo et al., 2013). The third cluster we designated proliferating (Prol)

193 cells, due to their high expression of CD69, IRF4, MYC and CD83 (Figure S3A).

195 DEGs were again identified in the same manner as with the Smart-seq 2 dataset. Visual inspection of a 196 heatmap showing the top DEGs combined with phylogenetic analysis (Figure 2B; Figure S2B)

197 suggested that the remaining 8 clusters could be grouped into 3 distinct "superclusters". Similar to the 3 198 clusters identified in our Smart-seq 2 analysis, GSEA showed that the 3 superclusters identified using 199 the 10x Chromium correspond to atBC, MBC and ABC populations (Figure 2C; Figure S3B). Notably 200 the $\mathrm{ABC}$ cells identified in the 10x Chromium analysis appeared to express a stronger $\mathrm{ABC}$ signature 201 than the "cluster 3" cells from the Smart-seq2 analysis. To determine the relationship between our 202 Smart-seq 2 clusters and those found using 10x Chromium, we combined both datasets using Seurat's 203 integration command (Figure S3C-D). This integrated dataset revealed that there was general 204 consensus between the atBC and MBC clusters identified separately using the Smart-seq 2 and 10x 205 Chromium methodologies. However only $20 \%$ of the "cluster 3" Smart-seq2 cells were found to be $206 \mathrm{ABCs}$ in the integrated dataset. Rather, these cells clustered more closely with the MBC1 207 subpopulation within the MBC supercluster (Figure S3C-D). 
209 Similar to the Smart-seq2 atBCs, cells in the 10x Chromium atBC "supercluster" showed higher 210 expression of atypical genes such as ITGAX, FCRL5, TBET, LILRB1 and CD19 (Figure 2D; Figure 211 S3B). The presence of the three sub-clusters (designated atBC1, atBC2 and atBC3) showed that there 212 was nonetheless some heterogeneity within this population, such as the lower expression of ITGAX, 213 FCRL5 and TBET in atBC2 and almost no expression of these markers in atBC3. In agreement with 214 observations seen in mouse models suggesting that these cells are primed for antigen presentation 215 (Rubtsov et al., 2015), we found that cells from the atBC supercluster upregulate genes associated with 216 antigen presentation and processing (Figure 2E). The MBC supercluster, made up of the sub-clusters 217 designated $\mathrm{MBC} 1, \mathrm{MBC} 2$ and $\mathrm{MBC} 3$, lacked a clear core gene signature with less than 10 positively expressed DEGs suggesting that these cells were in a state of quiescence. Consistent with the idea that these cells represent a recirculating, memory population, many were found to have high mRNA expression of the lymphoid homing receptors CCR7 and SELL (Figure 2F). The ABC super-cluster, made from 2 clusters ( $\mathrm{ABC} 1$ and $\mathrm{ABC} 2)$, had high expression of the activated $\mathrm{B}$ cell genes such as $C D 1 C$ and $C S K$ (Figure 2F). CXCR3 was not highly expressed in the ABC supercluster, but rather was most abundant on the $\mathrm{MBC} 1$ population, which is consistent with the fact that most of the Cluster 3 $C X C R 3$ expressing cells identified in the Smart-seq2 analysis map to this population.

Pseudotime Analysis reveals two distinct lineages of circulating B cells

228 To determine the lineage relationships between the distinct populations identified by unbiased 229 hierarchical clustering we used pseudotime analysis using the R package Monocle 3 (Trapnell et al., 230 2014). Visual inspection of the resulting UMAP appeared to reveal 2 distinct, major branches of 231 circulating B cells (Figure 3A). The first branch, made up of the more "classical" MBC2 and MBC3 232 memory populations, and $\mathrm{ABCs}$, had low progression along pseudotime (Figure 3A), indicating they 
were more closely related to naïve cells, which marked the beginning of pseudotime. Strikingly,

234 pseudotime appeared to form a loop, indicative of cells transitioning between states, such as activated

$235(\mathrm{ABC} 1$ and $\mathrm{ABC} 2)$ and quiescent $(\mathrm{MBC} 2$ and $\mathrm{MBC} 3)$ rather than forming terminally differentiated

236 populations. The second, more "atypical" branch consisting of the atBCs, MBC1 and some MBC3s,

237 had progressed further along pseudotime, suggesting they had differentiated further away from naïve

238 precursors than had the "classical" branch. Pseudotime could again be seen to form a loop, suggesting

239 that atBCs may also fluctuate between resting (MBC1 and MBC3) and activated (the atBCs) states. The

240 proliferating cells appeared to form their own distinct branch, or lineage, separate from either ABCs or

241 atBC. Interestingly, The PCs appeared entirely detached from the pseudotime pathway, indicating that

242 an intermediate PC population could not be found amongst circulatory B cells.

244 Similar results were obtained when we used a diffusion mapping approach to examine lineage

245 relationships. Two distinct branches of cells could be seen diverging from a central cluster of MBCs

246 (Figure 3B). atBCs formed the first branch with atBC2s and atBC3s predominantly found at the base of

247 the branch, closer to the MBCs and atBC1s found at the tip. The second branch was made of ABCs at

248 the base extending to PCs at the tip suggesting that following immunization or infection, MBCs may

249 follow either a classical activation pathway that ultimately leads to terminally differentiated antibody

250 secreting PCs or an "atypical" pathway, culminating in the formation of $\mathrm{Tbet}^{+} \mathrm{FCRL} 5^{+} \mathrm{CD} 11 \mathrm{c}^{+}$atBCs.

252 Atypical B cells are represented at high frequencies in all individuals, but are not necessarily CD27$253 C D 21^{-}$

255 Having identified 11 populations of circulating B cells by transcriptional profiling, we verified that all 256 populations could be found in all individuals tested, albeit in slightly different proportions (Figure 4A; 
257 Figure S4). The malaria-exposed donors had high numbers of atBC1 and atBC2 populations, but 258 surprisingly the non-exposed donors also had significant numbers of atBCs $(\sim 20 \%)$ that were largely

259 atBC3 (Figure 4A; Figure S4B). Non-exposed donors and malaria-exposed donors alike also

260 additionally carried high numbers of the MBC1 population $(\sim 20 \%)$ which appears related to the atBC

261 lineage (Figure 4A; Figure S4). This number of atBCs and related cells, as identified by transcriptomic

262 techniques, contrasts with previous flow cytometry analysis which shows that non-malaria exposed

263 healthy donors typically carry few (generally $<5 \%$ ) CD27-, CD21- atypical cells (Illingworth et al.,

264 2013; Weiss et al., 2009).

266 To address this discrepancy, we used CITE-seq to correlate the cell surface levels of our candidate 267 markers CD11c and CXCR3 as well as CD27 and CD21 to our transcriptomic data for each cell and 268 cluster (Figure 4B). CITE-seq data was exported into flow cytometry analysis software (FlowJo) for 269 further processing and presentation. CD11c was abundant on atBC1 and atBC2 cells which are 270 common in malaria exposed individuals but was only found at low levels on the atBC3 population 271 which was preferentially found in the non-exposed donors (Figure 4C). Among MBC populations we 272 found that $\mathrm{CD} 11 \mathrm{c}$ was more abundant on the $\mathrm{MBC} 1$ populations that appears related to atBC lineage. 273 Analysis of CD21 and CD27 expression showed that while atBC1 cells were almost exclusively CD21274 and $\mathrm{CD}_{27}$, the other populations of atBCs had more heterogeneous expression of these markers 275 (Figure 4D and E). Thus, the atBC3 population appears to be a cryptic atBC population which cannot 276 be detected via conventional flow cytometry strategies. These data may support the conclusion from 277 our pseudotime analysis that there is a spectrum of activation within atBCs, with $\mathrm{CD}^{-} 1^{-}, \mathrm{CD}^{2} 7^{-}$, $278 \mathrm{FCRL}^{+}, \mathrm{CD} 11 \mathrm{c}^{+} \mathrm{B}$ cells representing a more activated phenotype (atBC1 and 2) while other cells 279 become more quiescent, losing expression of these markers while retaining a core gene signature 280 (atBC3 and MBC1). 
282 We further used this analysis to investigate the utility of our markers for identifying MBC and ABC 283 populations. $\sim 50 \%$ of the $\mathrm{MBC} 1$ and $\mathrm{MBC} 2$ populations were $\mathrm{CD} 27^{+}, \mathrm{CD} 21^{+}$, but only $\sim 20 \%$ of the $284 \mathrm{ABC}$ populations resembled the activated $\mathrm{CD} 27^{+}, \mathrm{CD} 21^{-}$phenotype. $\mathrm{MBC} 3$ also looked somewhat 285 activated as $\sim 30 \%$ of cells were $\mathrm{CD} 27^{+} \mathrm{CD} 21^{-}$(Figure $4 \mathrm{D}$ and $\mathrm{E}$ ). This is consistent with the 286 observation that this population does appear to express some activation genes, albeit at low levels 287 (Figure 2F-Figure S2). As expected from our gene expression data CXCR3 was not a useful marker for the $\mathrm{ABC}$ populations, rather $\mathrm{CXCR} 3$ was most abundant on $\mathrm{MBC} 1$ cells consistent with the $C X C R 3$ expressing "cluster 3" cells from the Smart-seq2 data set mapping to this population. CXCR3 was also found on the atBC2 population which is consistent with this being a marker of "activated" atBCs.

Overall these data show that that CD27 and CD21 poorly mark different B cell memory subsets, however they suggest that - while imperfect - CD11c is a useful maker of the atBC lineage.

Subsets of circulating B cells do not segregate with Ig subclass or V region usage

In murine models different memory B cell subsets may be defined by their Ig-subclass, including in malaria infection (Krishnamurty et al., 2016; Pape et al., 2011). We therefore performed analysis of the VDJ and constant region usage of the heavy chains of our different B cells populations. The switched subclasses (IgM, IgG1, IgG2, IgG3, IgG4, IgA1, IgA2 and IgE) were found in all non-naïve B cell populations, with the exception of IgE, of which only 2 cells were found across all donors (Figure 5A).

301 The population we identified as naïve was largely $\operatorname{IgM}^{+}$with some cells expressing $\operatorname{IgD}^{+}$further supporting this designation. In both malaria-exposed donors, IgG3 was overrepresented in the atypical 
304 Adjei et al., 2017). Overall, these data suggest that the transcriptional signatures are distributed across 305 all Ig subclasses.

306

307 We further examined the variable region sequence of each cell to determine the level of SHM and 308 clonal relationships based on identical IGHV CDR3's with matching IGLV or IGKV. Similar to the 309 constant region, V region usage was similar between all donors and B cell clusters (Figure S5A-B). All 310 clusters, with the exception of the "naïve" cells showed significant SHM, confirming that these cells 311 were antigen experienced, post-GC B cells (Figure 5B). The degree of SHM differed significantly 312 based on the cell population and donor (Figure 5B). Notably across all populations, non-exposed 313 donors apparently carried lower levels of SHM than malaria-exposed donors, perhaps indicating lower 314 lifetime pathogen burden (Figure 5B). However, most V(D)J databases are based on Europeans and 315 may reflect allelic differences between populations of African and European ancestry. In 3/4 donors, 316 ABC2 and PCs had higher levels of SHM compared to either atBC populations or MBC populations 317 (Figure 5B-C), which may be consistent with these populations being related as indicated by diffusion mapping analysis (Figure 3C). Finally, 1-5\% of all BCRs sequenced were shared between 2 or more cells in each sample (Figure 5D). In all individuals, expanded clones could be found, in most cases these expanded clones were found within clusters, however clones could also be found shared between

321 clusters, including across superclusters indicating that a single clone can potentially adopt multiple cell 322 fates (Figure 5D).

326 To extend the analysis of circulating B cells beyond the original 4 donors, we performed flow 327 cytometry analysis of B cells from the PBMCs of 11 malaria-exposed and 7 non-exposed individuals 
328 (Table S1). Because the pseudotime analysis suggested that both classical and atypical lineages can

329 cycle between activated and resting states we also included CD71 as a marker of B cell activation

330 (Ellebedy et al., 2016). To identify atBCs we used a panel of CD11c, FCRL5, CD27, CD21 and T-bet,

331 though none of these markers would be likely to capture the "cryptic" atBC3 population found in non-

332 exposed healthy adults which did not express any obvious candidate surface markers based on our

333 transcriptomic and CITE-Seq analysis.

335 Consistent with our own transcriptomic and CITE-seq data as well as the data of others (Portugal et al., 336 2015; Weiss et al., 2009), we found that CD11 $\mathrm{c}^{+}$cells were considerably enriched in malaria-exposed 337 donors (Figure 6A-B). We were able to identify a small population $(\sim 5 \%)$ of $\mathrm{CD} 11 \mathrm{c}^{+} \mathrm{B}$ cells among the 338 non-exposed donors which were CD19 ${ }^{\text {hi }}$, CD20 $0^{\text {hi }}$ and FCRL5 ${ }^{\text {hi }}$, but only a few of these cells were

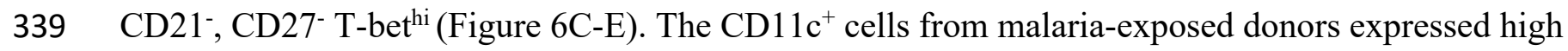
340 levels of T-bet and were mostly CD21- CD27- (Figure 6D-E). This supports the finding that there may

341 be a spectrum of atBCs, which may also explain why these cells have been identified and characterized 342 in slightly different ways in different pathologies (Jenks et al., 2018; Moir et al., 2008; Weiss et al., 343 2009). In both non-exposed and malaria-exposed donors around $30 \%$ of B cells were $\mathrm{CD} 71^{+} \mathrm{CD}_{11 \mathrm{c}^{-}}$ 344 (Figure 6A-B) which is consistent with the proportion of ABCs identified by single cell RNA-seq. 345 However, CD71 was also expressed on a high proportion of the CD11 $\mathrm{c}^{+} \mathrm{B}$ cells. This further supports 346 the observations from the pseudotime analysis that atypical cells may fluctuate between activated and 347 resting states. Further analysis found that these $\mathrm{CD} 71^{+}$atBCs expressed high levels of CXCR3 (Figure $3486 \mathrm{E}$ ), further supporting the observation from CITE-seq data that CXCR3 is a marker of activated 349 atBCs. 
353 Our pseudotime analysis indicated that the majority of circulating B cells can be separated into two

354 distinct lineages, classical or atypical, with cells in each lineage able to fluctuate between activated and

355 resting states. While the diffusion map results suggest that the classical lineage gives rise to antibody

356 secreting PCs, the role of the atypical lineage in the immune response is still not understood. In the past

357 it has been suggested that atBC are an exhausted or dysfunctional cell population that arise following

358 chronic antigen exposure (Portugal et al., 2015; Sullivan et al., 2015). We therefore wanted to examine

359 a situation in which we could (i) track antigen specific cells after primary exposure and (ii) continue to

360 follow those cells upon antigen re-exposure. We would thus be able to determine the point in an

361 immune response at which atBCs arise as well measure their longevity and their capacity to be recalled.

362 To meet these criteria, we tracked B cells specific for PfCSP in a cohort of malaria-naïve individuals

363 who were given three doses of a whole $P$. falciparum sporozoite vaccine (PfSPZ) at 8-week intervals

364 (Ishizuka et al., 2016; Lyke et al., 2017). These sporozoites are irradiated so do not establish ongoing

365 infection, though there is previous evidence of antigen persistence (Cockburn et al., 2010). Blood

366 samples were obtained at the time of each vaccination and $1 \mathrm{wk}$ and $2 \mathrm{wks}$ post each vaccination and

367 flow cytometric analysis was performed to identify and characterize PfCSP-specific B cells (Figure 7A;

368 Figure S6A). We were further able to identify PfCSP specific PCs in these individuals (Figure S6A).

370 By 1 week after immunization the number of CSP specific B cells had begun to increase, though most

371 remained $\mathrm{CD}^{-} 1^{-}$, CD11 $\mathrm{c}^{-}$(Figure 7A-C). However, by 2 weeks post immunization, significant

372 populations of $\mathrm{CD} 71^{+}$and $\mathrm{CD} 11^{+} \mathrm{B}$ cells could be seen, with many of the $\mathrm{CD} 11 \mathrm{c}^{+} \mathrm{B}$ cells co-

373 expressing CD71, matching those seen in malaria-exposed individuals (Figure 7A-C). By 8 weeks post

374 immunization distinct $\mathrm{CD} 71^{+}$and $\mathrm{CD} 11 \mathrm{c}^{+} \mathrm{B}$ cell populations were seen, but the $\mathrm{CD} 11 \mathrm{c}^{+} \mathrm{B}$ cells no

375 longer expressed high levels of CD71 further supporting the idea that this is a marker of recent 
activation on atBCs (Figure 7C). Importantly, both $\mathrm{CD} 71^{+}$and $\mathrm{CD} 71^{-} \mathrm{CD} 11 \mathrm{c}^{+} \mathrm{B}$ cells expressed high

377 levels of CD19, CD20 and low levels of CD21 and CD27 further supporting the fact that these cells represent a bona-fide atBC population (Figure S6B-C).

We further tracked these B cell populations following boosts after 8 and 16 weeks (Figure 7A-C). response did not change at the second boost. During the first boost the $\mathrm{CD}^{+} 1^{+} \mathrm{CD} 11 \mathrm{c}^{-}$population peaked after 1 week; more rapidly than during the primary response (Figure $7 \mathrm{C}$ ), however the total

$\mathrm{CD} 11 \mathrm{c}^{+}$population continued to expand and only peaked 2 weeks after each boost. Notably the kinetics of the atBC population are distinct from those of the PC population which is only detectable 1 week after each boost, which further suggests that in normal conditions atBCs are not pre-PCs. Finally, 16 weeks after the final boost (wk 34) we examined the number and phenotype of the different cell populations. At this "memory" timepoint most cells were double negative, but residual populations of CD1 $1 \mathrm{c}^{+}$and $\mathrm{CD}^{+} 1^{+} \mathrm{B}$ cells could still be detected (Figure 7A-C).

Finally, we wanted to know if our findings could extend beyond Plasmodium to another vaccination setting, also involving acute exposure to antigen. We therefore examined how these cells responded in a recall response to the inactivated influenza vaccine (IIV) (Koutsakos et al., 2018). Using recombinant rHA probes to two IIV antigens: A/California/07/09-H1N1 (A/Cal09-H1) or B/Phuket/3073/2013 of subjects prior to immunization (Figure 7D; Figure S6D). $\mathrm{CD}^{+} 1^{+} \mathrm{ABCs}, \mathrm{CD} 11 \mathrm{c}^{+}$atBCs and double negative MBCs to both influenza antigens could be found at baseline in most individuals as expected, reflecting past exposure to influenza virus infection or vaccination (Figure 7D-F). Following 


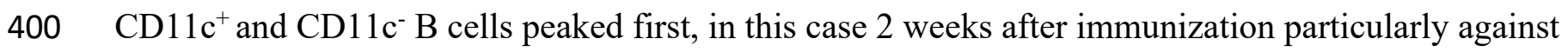
401 the B/Phuket-HA strain, while CD11 ${ }^{+} \mathrm{B}$ cells had a more sustained expansion (Figure 7D-F). Again, 402 influenza specific CD11 $\mathrm{c}^{+}$cells found after vaccination expressed high levels of CD19, CD20 and 403 FCRL5, and high proportions of these cells were CD21- and CD27- consistent with them being an 404 atypical population (Figure S6E-F). All together these data reveal that atBC arise during the primary 405 response and that these cells appear to participate in recall responses upon re-exposure to antigen, 406 countering the suggestion that these cells are a dysfunctional or exhausted population. 


\section{Discussion}

410 Atypical B cells, which have been conventionally defined based on specific cell surface markers have

411 been found in excess in many pathological conditions, in particular chronic or repeating infections and

412 autoimmunity. This association with disease, along with the difficulty of re-stimulating these cells in

413 vitro has led to the assumption that these are an exhausted or even pathological population. Our

414 observations using single cell transcriptional analysis that atBC are more abundant than expected in

415 both healthy non-exposed and malaria-exposed individuals lead us to test the hypothesis that these cells

416 are a stable lineage that arise in response to antigenic stimulation. Accordingly, we tracked these cells

417 in controlled conditions of vaccination and further showed that these cells arise during the primary

418 immune response and can be recalled normally on multiple re-exposures. Importantly these cells are

419 not merely recently activated B cells as they have a distinct gene signature from previously described

420 ABCs. Thus, while - in accordance with previous literature - we have used the term "atypical" to

421 describe this lineage, the data presented here suggests that atBCs contribute to antigen-specific primary

422 and recall antigen specific responses, and thus are a typical part of the B cell response to antigen.

423

424 It has been hypothesized that atBCs are exhausted (Portugal et al., 2015; Sullivan et al., 2015) or

425 recently activated cells (Jenks et al., 2018; Perez-Mazliah et al., 2018). However, our observations that

426 atBC are generated during the primary response to sporozoite vaccination and can be recalled following

427 booster immunizations, either to sporozoite or influenza antigens would suggest that atBC are not

428 necessarily exhausted cells and can in fact participate in normal immune responses. Furthermore, our

429 single cell RNA-seq data clearly differentiates atBC from previously described ABCs. Nonetheless

430 there is also evidence that atBCs themselves can be activated which may resolve some of the

431 discrepancies seen in the literature. Specifically, we have shown that $\mathrm{CD} 71^{+}$atBC appear early in the 
432 response and abate quickly, giving rise to a more conventional $\mathrm{CD} 71^{-}$atBC population. These $\mathrm{CD}^{+} 1^{+}$

433 atBCs likely represent a population of recently recalled cells. Interestingly, while the first study to

434 describe ABCs cells used CD71 as the primary marker for ABC (Ellebedy et al., 2016), CD11 $\mathrm{c}^{+} \mathrm{B}$

435 cells, which we have shown can express CD71 were not excluded. Thus, this bulk sorted population

436 may have contained some atBCs which may explain why in our smaller Smart-seq2 dataset, the atBC

437 cluster was found to be enriched for ABC genes. We did however observe that the antigen-specific

438 atBC population does diminish overtime in our sporozoite vaccinated individuals. One explanation for

439 this is that atBCs are not a bona fide memory population that are as long-lived as the MBCs. However,

440 the presence of a sizeable CD11 $\mathrm{c}^{-} \mathrm{FCLR}^{-} \mathrm{CD} 27^{+}$atBC (atBC3) as well as MBC1 populations in

441 healthy non-exposed individuals suggests that there may exist a pool of "cryptic", quiescent MBCs

442 primed to differentiate into $\mathrm{CD} 11 \mathrm{c}^{+}$atBCs following re-exposure.

444 We initially described a tripartite division in our description of the circulating B cell subsets. This is

445 based on the unsupervised clustering of our single cell RNA-seq datasets revealing distinct

446 "superclusters" of circulating B cells: Activated (ABC), Memory (MBC) and Atypical (atBC).

447 However, an alternative classification informed by pseudotime analysis and our data from vaccination

448 cohorts would suggest a division of two distinct lineages, or pathways of MBCs and their

449 corresponding activated populations. For example, in the classical pathway, MBC2 are quiescent cells

450 that, following antigenic stimulation, transition into proliferating ABCs that can go on to either

451 terminally differentiate into PCs or reseed the MBC pool. A previous study showed that up to $60 \%$ of

$452 \mathrm{ABC}$ clones sequenced at day 7 could be found in the $\mathrm{MBC}$ subset 90 days post vaccination, revealing

453 that $\mathrm{ABC}$ can downregulate their activation markers and become MBCs over time (Ellebedy et al.,

454 2016). Under this model the $\mathrm{ABC} 2$ and $\mathrm{MBC} 3$ populations likely represent a spectrum of cells that

455 were either recently activated or entering a state of quiescence, rather than defined B cell fates. 
456 Interestingly, our VDJ analysis revealed that B cell clones could be found in both cell lineages,

457 indicating that the progeny of a single B cell may adopt both cell fates

458

459 While our data show that atBC are part of a normal B cell response, the function of these cells remains 460 elusive. Early studies revealed that it was difficult to differentiate atBCs into antibody secreting cells 461 under standard conditions in vitro (Moir et al., 2008; Portugal et al., 2015; Sullivan et al., 2015).

462 However bulk RNA-seq analysis of atBC-like cells from SLE patients showed higher expression of 463 genes associated with PC maintenance in this populations lead to the hypothesis that atBCs represent a 464 precursor PC population (Jenks et al., 2018). Our analysis however, using single cell RNA sequencing 465 techniques, could not find any evidence of these genes being upregulated in any of our atBC 466 populations. We also observe that our atBC population in sporozoite vaccinated individuals continues 467 to expand even 2 weeks after booster immunization while PC populations peaked 1-week post468 immunization. A reconciliation of these conflicting results may be that in pathogenic conditions such as 469 SLE, atBCs can be driven to become pathogenic antibody secreting cells. Consistent with this the 470 TLR7 pathway is implicated in SLE development and it has been found that including TLR7 agonists 471 within the stimulating condition can help differentiate atBCs into PCs (Jenks et al., 2018; Perez472 Mazliah et al., 2018; Rivera-Correa et al., 2019; Rubtsova et al., 2013).

474 A potential role for atBC may be specifically in the clearance of viral infection, as research in murine 475 models has found that $\mathrm{T}-\mathrm{bet}^{+} \mathrm{CD} 11 \mathrm{c}^{+} \mathrm{B}$ cells, are required for effective clearance of viral infection 476 (Barnett et al., 2016; Rubtsova et al., 2013). Our study has focused on relatively inflammatory B cell 477 stimuli such as malaria infection and attenuated pathogen vaccines. It may be that atBC are 478 preferentially formed in these conditions rather than subunit vaccination in less inflammatory adjuvants 479 such as alum and thus would be expected to play a role in control of infection. Finally, it has been 
480 proposed that atBCs are potent antigen presenting cells (Rubtsov et al., 2015). In agreement with this

481 we did find that human atBC do appear to have higher expression of MHC II as well as the co-

482 stimulatory molecule CD86 and components of the MHC Class II antigen processing pathway,

483 indicating that they present more antigen then other B cell types. B cells have recently been shown to

484 be required for the priming of Tfh cells in the context of malaria infection, though the role of

485 individuals subsets was not studied (Arroyo and Pepper, 2020). Intriguingly another mouse study also

486 revealed that ablation of $\mathrm{CD} 11 \mathrm{c}^{+} \mathrm{B}$ cells lead to the collapse of germinal centers after one

487 immunization (Baumjohann et al., 2013). Thus, these data suggest that atBCs could represent a

488 population of specialized antigen presenters, although further investigation is needed to confirm this

489 hypothesis.

490

491 Here we provide an atlas of the B cell subsets circulating in human blood. Our powerful single-cell

492 RNA-seq analysis combined with CITE-seq technologies and VDJ profiling enables us to reconcile our

493 data with existing classifications of B cell memory based on flow cytometry markers or Ig-subclass.

494 We have been able to resolve some key controversies in the human B cell literature, most notably by

495 finding that atBCs are more abundant than previously expected in healthy donors and showing that

496 these cells can be induced by primary exposure to acute antigen. Thus, our data suggests that atBC are

497 a critical and typical component of the humoral immune response. 


\section{Author Contributions}

499

500 Conceptualization (HJC, RA, AHI, FMN, IAC). Data curation (HJC, XL, TDA, FMN, IAC). Formal

501 Analysis (HJC, RA, XL, XG, TDA, FL). Funding acquisition (KK, RAS, FMN, IAC). Project

502 Administration (KEL, SLH) Investigation (HJS, RA, AHI, RV, EN, OK, JM, MK, THON, MN, PM, 503 AAB, NK, SC). Methodology (AE, FL). Resources (AKW, SJK, FL, KK, RAS, FMN). Software (FL). 504 Supervision (SJK, FL, KK, RAS, FMN, IAC). Visualization (HJS, XL, IAC). Writing - original draft 505 (HJS, IAC). Writing - review \& editing (FL, KK, RAS, FMN).

Acknowledgements

509

510 This work was supported by start-up funds from the Australian National University to I.A.C. and 511 NHMRC project grant support to I.A.C. (GNT1158404). We would like to thank Harpreet Vohra and 512 Michael Devoy of the Imaging and Cytometry Facility at the Australian National University for 513 assistance with flow cytometry and sorting. We also thank the staff of the biomolecular resource 514 facility at the John Curtin School of Medical Research for assistance with single cell RNA-seq. 515 Production and characterization of PfSPZ Vaccine were supported in part by National Institute of 516 Allergy and Infectious Diseases Small Business Innovation Research Grants 5R44AI055229-11 (to 517 S.L.H.), 5R44AI058499-08 (to S.L.H.), and 5R44AI058375-08 (to S.L.H.). We would like to thank the 518 University of Maryland study volunteers from malaria clinical trial VRC314. We are grateful to the $519 \mathrm{KEMRI/CGMRC} \mathrm{field} \mathrm{team} \mathrm{for} \mathrm{their} \mathrm{dedication} \mathrm{in} \mathrm{the} \mathrm{recruitments,} \mathrm{malaria} \mathrm{surveillance} \mathrm{data} \mathrm{and}$ 520 sample collection, and the laboratory team that processed the samples. 
521 We are also indebted to the study participants. FMN was supported by an MRC/DFID African

522 Research Leadership Award (MR/P020321/1), a Senior Fellowship from EDCTP (TMA2016SF-1513)

523 and the samples were collected within the Kilifi immunology cohorts supported by various Wellcome

524 grants. RA was supported through the DELTAS Africa Initiative [DEL-15-003]. The DELTAS Africa

525 Initiative is an independent funding scheme of the African Academy of Sciences (AAS)'s Alliance for

526 Accelerating Excellence in Science in Africa (AESA) and supported by the New Partnership for

527 Africa's Development Planning and Coordinating Agency (NEPAD Agency) with funding from the

528 Wellcome Trust [107769/Z/10/Z] and the UK government. This manuscript is published with

529 permission from the Director, KEMRI.

530

531

\section{Conflict of interest statement}

533

534 S.C., N.K., B.K.L.S., and S.L.H. are salaried employees of Sanaria Inc., the developer and owner of 535 PfSPZ Vaccine and the investigational new drug (IND) application sponsor of the clinical trials. S.L.H. 536 and B.K.L.S. have a financial interest in Sanaria Inc. All other authors declare no conflict of interest. 


\section{Materials and Methods}

\section{8}

539

Human samples and ethics statement

540 All research was conducted according to the principles of the Declaration of Helsinki, which included

541 the administration of informed consenting in the participant's local language. Studies in Australia were

542 further performed in accordance with the Australian National Health and Medical Research Council

543 (NHMRC) Code of Practice.

545 The malaria-immunology cohort and vaccination studies, under which the samples described were 546 collected in Kenya, were approved by the Kenyan Medical Research Institute Scientific and Ethics

547 Review Unit, Nairobi, and the use of these samples at the Australian National University was further 548 approved by the Australian National University Human Research Ethics Committee (protocol number 549 2014/102). The Kenyan adults are members of the KEMRI/Wellcome Research Programme's 550 longitudinal malaria immunology cohort studies in Junju and Ngerenya villages (supplementary table 551 1), $20 \mathrm{~km}$ apart from each other in Kilifi, Kenya.. In addition, we included samples from the 552 RTS,S/AS01 phase 3 clinical trial. Blood was also drawn from healthy control Australian donors who 553 were recruited at the Australian National University.

555 VRC 314 clinical trial (https://clinicaltrials.gov/; NCT02015091) was an open-label evaluation of the 556 safety, tolerability, immunogenicity and protective efficacy of PfSPZ Vaccine. Subjects in the high 557 dose cohort received a total of three doses of $9 \times 10^{5}$ PfSPZ intravenously at week 0,8 and 16 . Blood 558 was drawn at the time of each immunization, as well as $7 \mathrm{~d}$ and $14 \mathrm{~d}$ after each immunization. Plasma 559 and PBMCs were isolated from all samples at these timepoints. Full details of the study are described 560 in (Lyke et al., 2017). 
562 The investigation of B cell responses after IIV immunization was approved by the University of

563 Melbourne Human Ethics Committee (ID 1443389.3) and the Australian Red Cross Blood Service 564 (ARCBS) Ethics Committee (ID 2015\#8). PBMCs were used from 8 donors taken on the day of IIV 565 immunization, as well as 14 and 28 days later. Full details of the study are described previously 566 (Koutsakos et al., 2018).

Flow cytometry

569 For samples from Kenya and the IIV cohort PBMCs were thawed and washed in PBS with $2 \%$ heat570 inactivated FBS. Cells were then stained with Live/Dead dye for 5 min in PBS before incubation with 571 fluorescently labelled antibodies for a further $30 \mathrm{~min}$. Details of all antibodies used are given in Table 572 S2. Flow-cytometric data was collected on a BD Fortessa or X20 flow cytometer (Becton Dickinson) 573 and analyzed using the software FlowJo (FlowJo). A BD FACs Aria I or II (Becton Dickinson) was 574 used for sorting cells.

575

576 For VRC314 clinical trial specimens PBMCs were thawed into prewarmed RPMI media then washed 577 with PBS. Cells were stained with Live/Dead dye for 15 minutes, washed in PBS with 2\% heat 578 inactivated FBS, and labelled with antibodies for an additional 30 minutes. Labelled cells were washed 579 with PBS 2\% FBS and fixed for 15 minutes in $0.5 \%$ PFA before and final wash and resuspension in 580 PBS 2\% FBS. Flow-cytometric data was collected on a BD X50 flow cytometer (Becton Dickinson) 581 and analyzed using the software FlowJo (FlowJo).

582

583 Tetramer Preparation 
584

\section{5}

586

587

588 589

Pf MSP1, AMA1 and TT were biotinylated with the Sulfo-NHS-LC-Biotinylation Kit (ThermoFisher) at a ratio of $1: 1$ according to the manufacturer's instructions, biotinylated (NANP) 9 repeat region of $P f$ CSP was sourced from Biomatik (Ontario, Canada). Biotinylated antigens were incubated with premium-grade SA-PE and SA־APC (Molecular Probes) or SA-BV421 and SA-BB660 (Biolegend and BD Horizon) at a molar ratio of 4:1, added four times with 15 min incubation at room temperature.

\section{Single cell RNA'seq using Smart-seq2}

Antigen-specific single cell RNA sequencing was performed using a Smart-seq 2 protocol (Picelli et al., 2014) with the following modifications. Cells were sorted into plates with wells containing $1 \mu 1$ of the cell lysis buffer, $0.5 \mu \mathrm{l} \mathrm{dNTP} \operatorname{mix}(10 \mathrm{mM})$ and $0.5 \mu \mathrm{l}$ of the oligo-dT primer at $5 \mu \mathrm{M}$. We then reduced the amount reagent used in the following reverse-transcription and PCR amplification step by half. The concentration of the ISPCR primer was also further reduced to $50 \mathrm{nM}$. Due to the low transcriptional activity of memory B cells, we increased the number of PCR cycles to 28. cDNA was then purified with AMPure XP beads at a bead to sample ratio of 0.8:1. Sequencing libraries were prepared using the Nextera XT Library Preparation Kit with the protocol modified by reducing the original volumes of all reagents in the kit by $1 / 5^{\text {th }}$. Another round of bead cDNA bead purification was preformed using a bead to sample ratio of $0.6: 1$. Sequencing was performed on the Illumina NextSeq sequencing platform. Following sequencing, fastq files were passed through the program VDJPuzzle (Rizzetto et al., 2018) where reads were trimmed using Trimmomatic, then aligned to the human reference genome GRCh37 using tophat2. Gene expression profiles were then generated using cufflinks 2. As a further QC step, cells where reads were mapped to less than $30 \%$ of the reference genome were removed. All following downstream analysis for transcriptomic data was performed using Seurat. Details of all key reagents for single cell RNA-seq are given in Table S3. 
609 Post-sorting, CD19+ CD20+ IgD $^{-}$B cells were incubated with Total-seq C antibodies (Biolegend) for 30 610 min and washed 3 times. The number of cells were then counted and 14000 cells per sample were run 611 on the 10X Chromium (10X Genomics). Library preparation was completed by Biomedical Research 612 Facility (BRF) at the JCSMR following the recommended protocols for the Chromium Single Cell 5' 613 Reagent Kit as well as 5' Feature Barcode and V(D)J Enrichment Kit for Human B cells. Libraries 614 were sequenced using the Illumina NovaSeq6000 (Illumina). The 10X Cell Ranger package (v1.2.0, 615 10X Genomics) was used to process transcript, CITE-seq and VDJ libraries and prepare them for 616 downstream analysis. Details of all key reagents for single cell RNA-seq are given in Table S3.

\section{Quantification and statistics}

\section{$621 \quad$ Single Cell RNA-seq analysis}

622 The package Seurat (version 3.1) (Butler et al., 2018) was used for graph-based clustering and 623 visualizations. All functions described are from Seurat or the standard R package (version 3.60) using 624 the default parameters unless otherwise stated. Each sample was initially analyzed separately using the 625 following procedures. Cells that expressed less than 200 genes and genes that were expressed in less 626 than 3 cells were excluded, along with cells that had greater than $10 \%$ mitochondrial genes. Gene 627 expression was normalized for both mRNA and CITE-seq assays using the NormalizeData function, 628 then the 2000 most variable genes for each sample were identified using FindVaraibleFeatures. Next 629 expression of all genes was scaled using ScaleData to linearly regress out sources of variation.

630 Principal component analysis on the variable genes identified above was then run with RunPCA. Based 631 on ElbowPlot results we decided to use 13, 20, 12 and 20 principal components (PCs) for the clustering 
632 of samples Non-Exp 1, Non-Exp 2, Exp 1 and Exp 2 respectively using FindNeighbours. FindClusters

633 was then run to identify clusters for each sample, using the resolutions $.3, .4, .5$ and .4 respectively.

634 FindAllMarkers was then used to identify clusters of non-B cells. The remaining cells in each sample

635 were then normalized and scaled again as above. Australian and Kenyan samples were combined

636 together first using FindIntegrationAnchors and then Intergratedata to create two combined datasets, 1

637 with both non-exposed samples and one with both malaria-exposed samples. These two combined

638 samples were further combined using the commands to from one combined dataset containing all 4

639 samples. The combined dataset was then scaled again as above and a PCA was run. Using FindClusters

640 with a resolution of 0.8 , we identified our 11 clusters. DEGs were identified using FindAllMarkers.

641 The clustering was visualized with Uniform Manifold Approximation and Projection (UMAP)

642 dimensionality reduction using RunUMAP and plotted using DimPlot with umap as the reduction.

643 Phylogentic analysis was done using BuildClusterTree to report the hierarchical distance matrix

644 relating an 'average' cell from each cluster. Log-normalized gene expression data was visualized using

645 violin plots (VlnPlots) as well as onto -UMAP plots (FeaturePlot). Heatmaps were generated using

646 DoHeatmap.

647 For Smart-seq2 analysis, cells with greater than 10\% mitochondrial genes were not excluded. 8 PCs

648 were used as determine by ElbowPlot. For clustering a resolution of 0.8 was used.

\section{Diffusion Map Analysis}

651 To create the diffusion map we utilized the R package destiny (Angerer et al., 2016). Our Seurat object 652 was converted into a SingleCellExperiment object using as.SingleCellExperiment. The diffusion map 653 was then generated using Destiny's DiffusionMap command.

654

655 Pseudotime Analysis 
656 Pseudotime analysis was preformed using the R package Monocle 3. Our Seurat Object was converted 657 into monocle3 main data calls cell_data_set. The default Monocle 3 workflow was then followed.

658

659

660 GSEA was done using javaGSEA through the Broad Institute. For each comparison, DEGs were 661 ranked by log-fold change and pre-ranked analysis using 1000 permutations was used to examine 662 enrichment in selected gene sets (SUPP table?).

663

664 VDJ Analysis

665 To determine the antigen-specific BCR repertoire, we made use of VDJpuzzle (Rizzetto et al., 2018) to 666 reconstruct full-length heavy and light chains from each cell from out Smart-seq2 dataset. From this we 667 were able to determine $\mathrm{V}$ region usage and mutation frequency.

668 VDJ sequences from the 10x dataset were obtained using the cellranger vdj command. From this 669 output, V region usage and mutation frequency could be determined.

670

671 Statistical Analysis

672 Statistical analysis of flow cytometry data was performed in GraphPad Prism for simple analyses 673 without blocking factors; all other analyses was performed in R (The R Foundation for Statistical 674 Computing) with details of statistical tests in the relevant figure legends. Abbreviations for $p$ values are 675 as follows: $\mathrm{p}<0.05=*, \mathrm{p}<0.01=* *, \mathrm{p}<0.001=* * *, \mathrm{p}<0.0001=* * * *$; with only significant $\mathrm{p}$ 676 values shown.

677

678 Data Deposition 
bioRxiv preprint doi: https://doi.org/10.1101/2020.05.28.120808; this version posted May 28, 2020. The copyright holder for this preprint (which

was not certified by peer review) is the author/funder, who has granted bioRxiv a license to display the preprint in perpetuity. It is made available under aCC-BY-NC-ND 4.0 International license.

679 Single cell RNA-seq data are deposited at NCBI BioProject accession number PRJNA612353:

680 https://dataview.ncbi.nlm.nih.gov/object/PRJNA612353?reviewer=bf7ee45b186vstua0d23qud1nk 
a

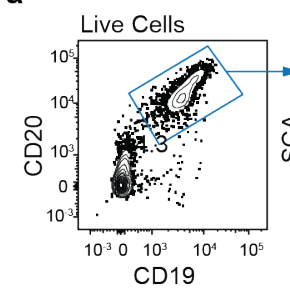

c

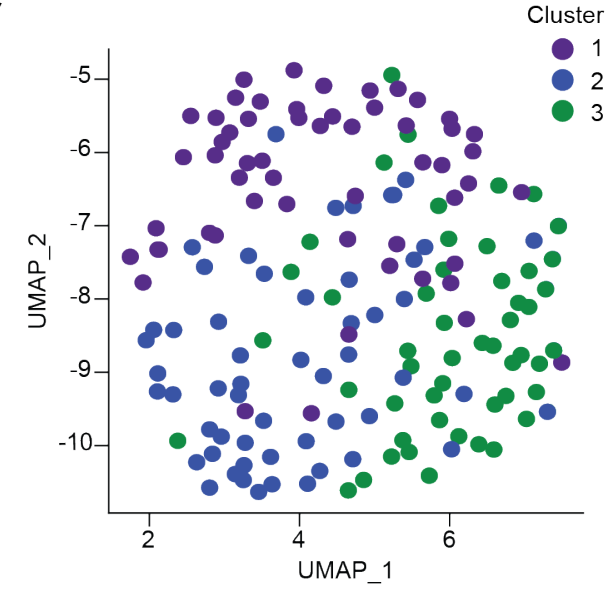

e

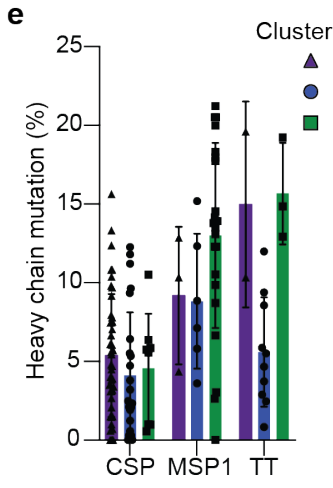

2way ANOVA $\mathrm{p}(\mathrm{Ag})=<0.0001$ $\mathrm{p}$ (cell type $)=0.0007$
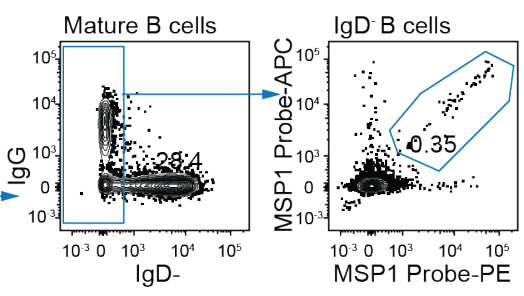

d
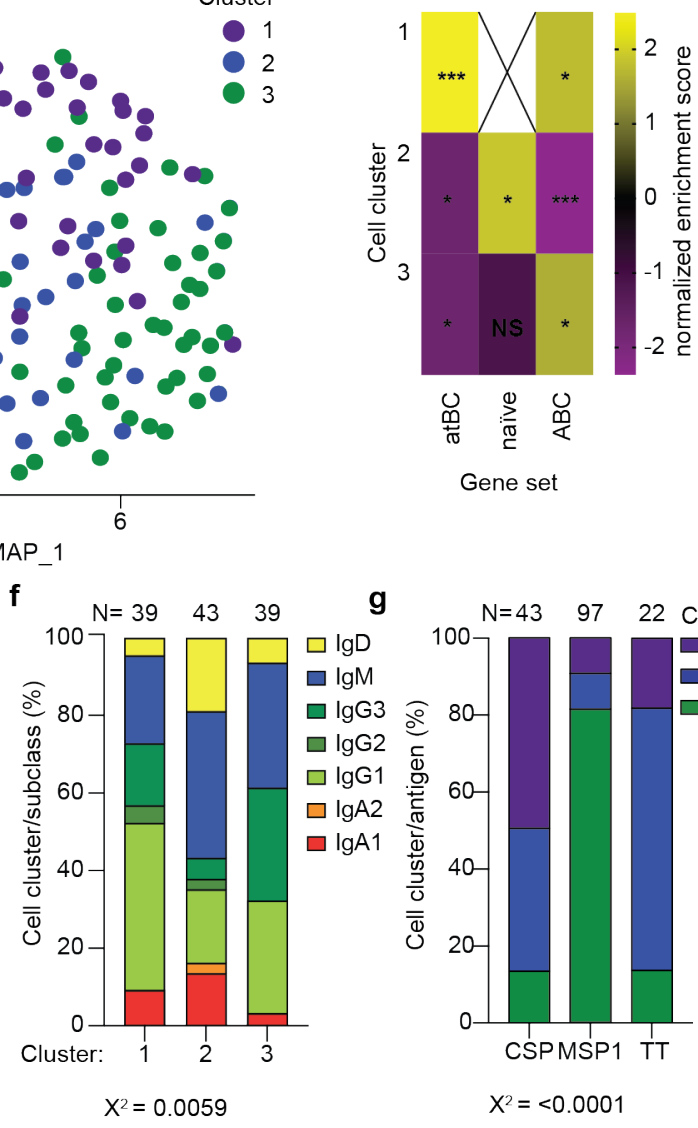

g
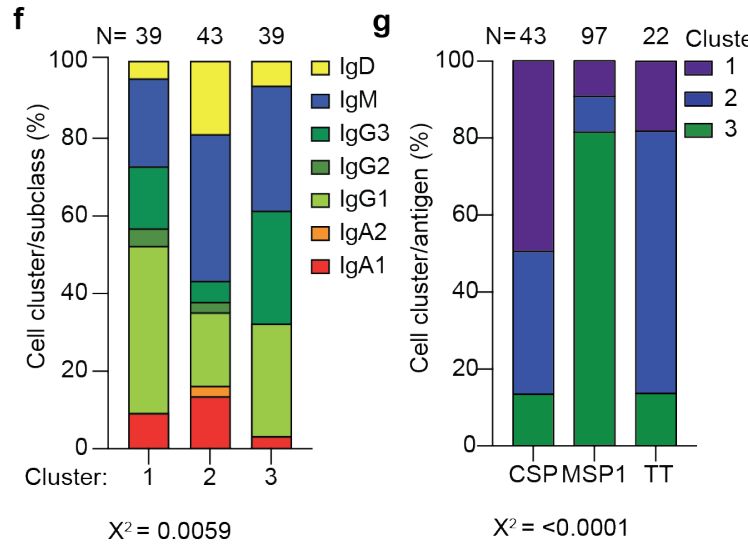

$X^{2}=<0.0001$ b

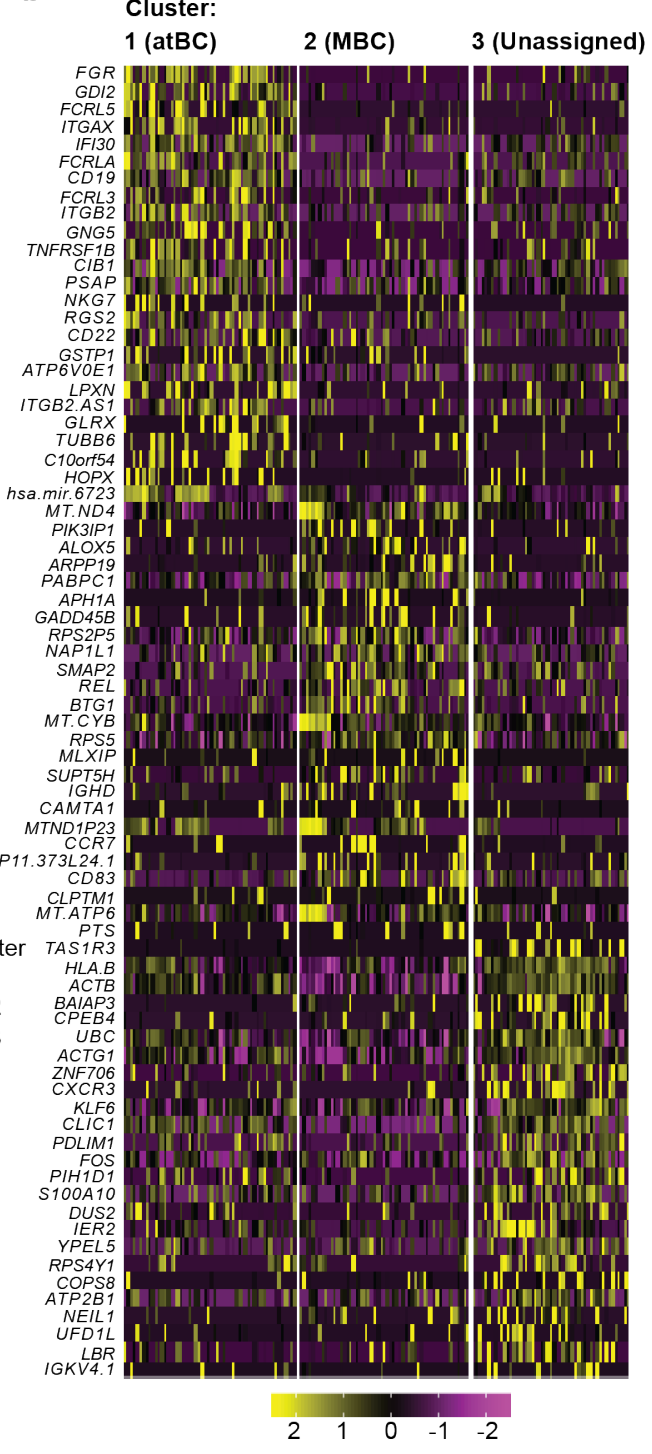

Cluster:

1 (atBC) 2 (MBC) 3 (Unassigned)

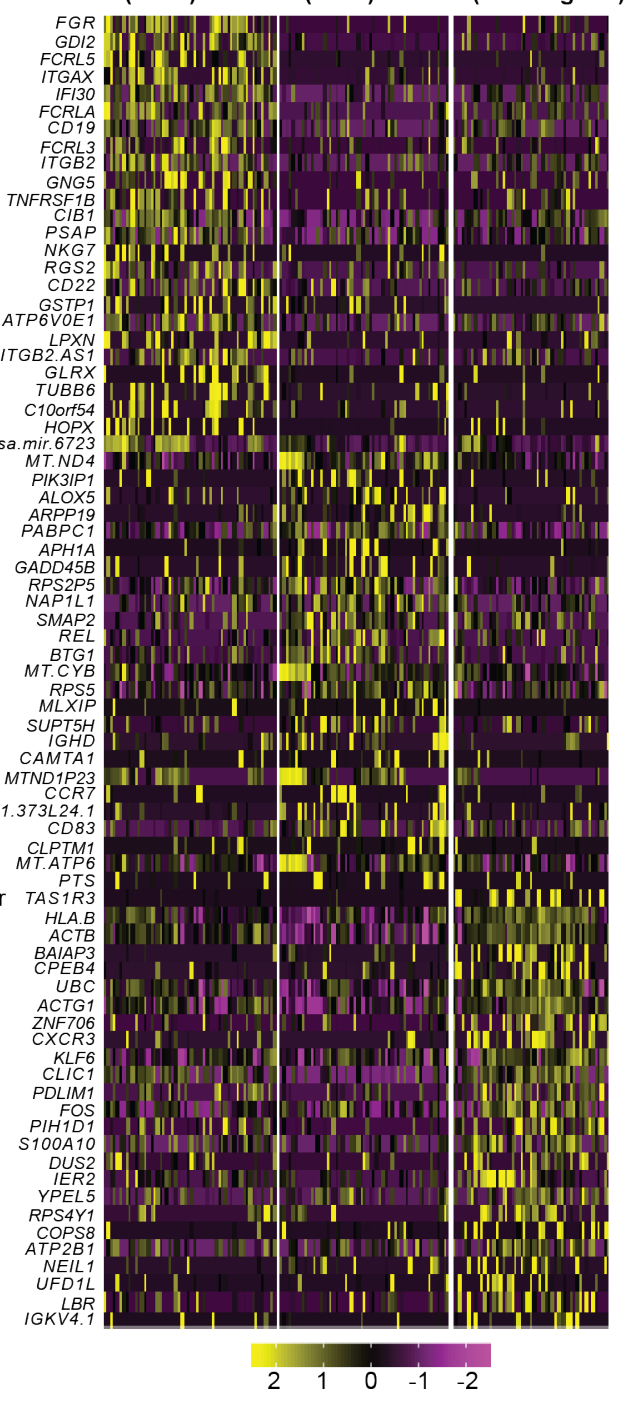

681

Figure 1: Three distinct populations of antigen-experienced B cells revealed by single cell RNAseq CSP, MSP1 and TT specific B cells were index, single cell sorted from malaria vaccinated and exposed donors; transcriptomic information for each cell was generated using Smart-seq2 methodology. A. Representative flow cytometry plots showing the gating strategy used to sort mature IgD- antigen-specific B cells. B. Heatmap showing the expression of the top 25 DEGs (row) per cluster for each cell (column). C. Unsupervised clustering of circulating antigen-specific B cells visualized using UMAP. Each point represents a cell and is colored by cluster. D. Heatmap displaying the normalized enrichment scores of multiple GSEA comparing each cluster vs previously published gene sets from atBCs, naïve B cells and ABCs E. Percentage of antigen-specific cells that were found in each cluster, analysis was by chi-squared test on the absolute values which are given above each bar. F. Percentage of antibody isotype usage by each cluster, analysis was by chi-squared test on the absolute values which are given above each bar. G. Percentage of mutations found in the heavy chain V(D)J region of each antigen-specific cell per cluster, analysis was by 2-way ANOVA including each subject 
a

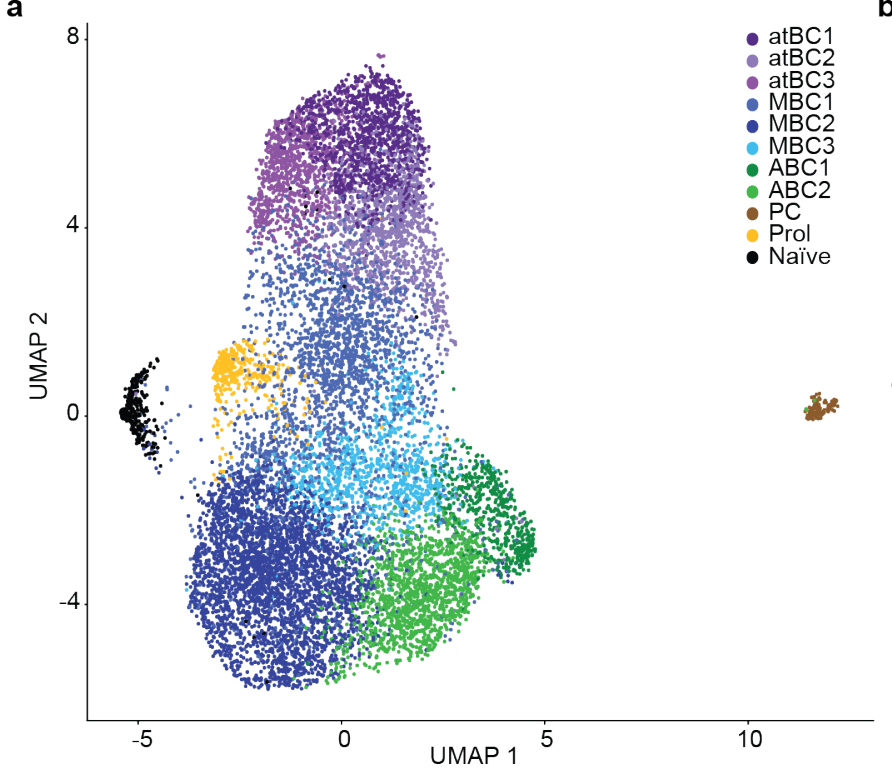

b

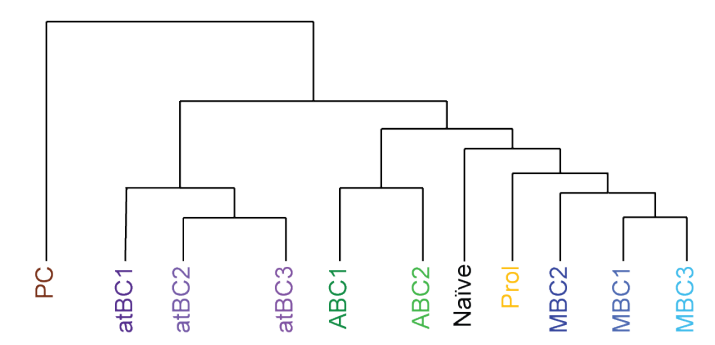

$\mathbf{C}$

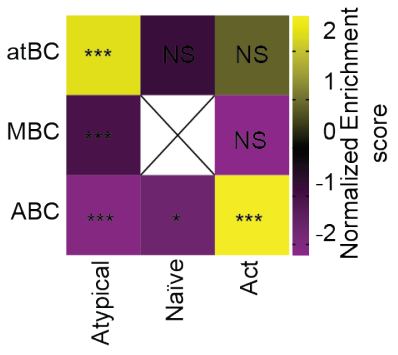

d Atypical B cell genes

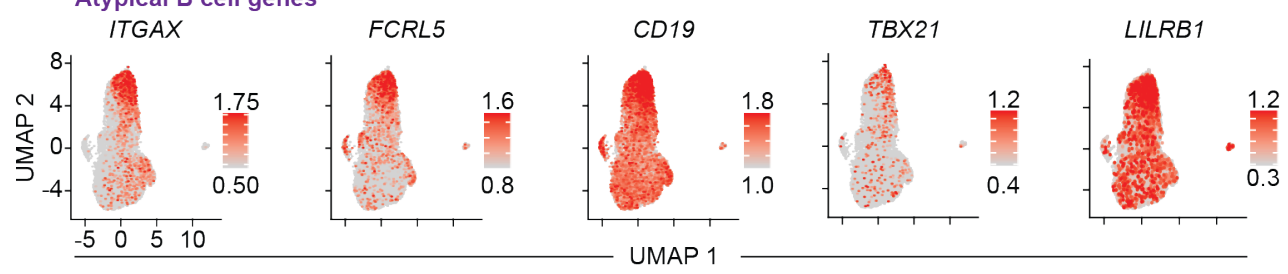

e Antigen presentation and processing genes

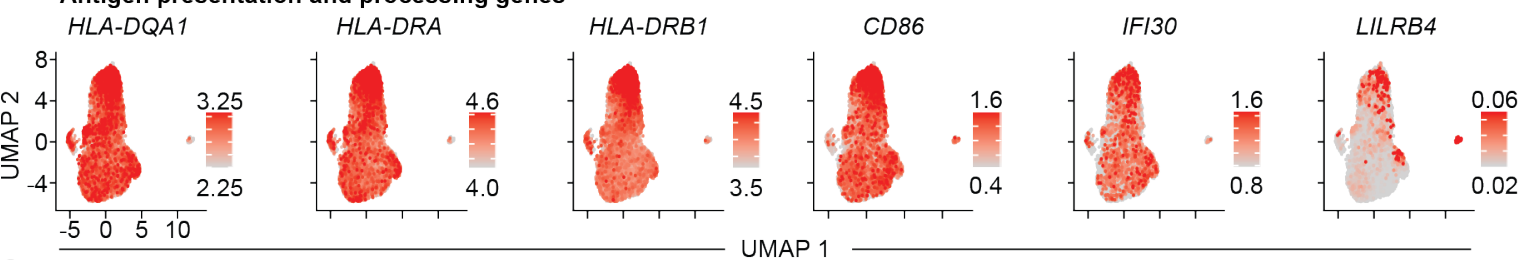

f

Memory B cell genes CCR7 SELL Activated $B$ cell genes
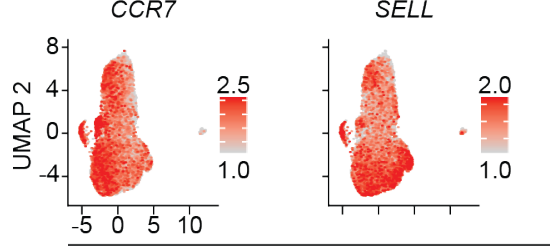
CXCR3

CD1C
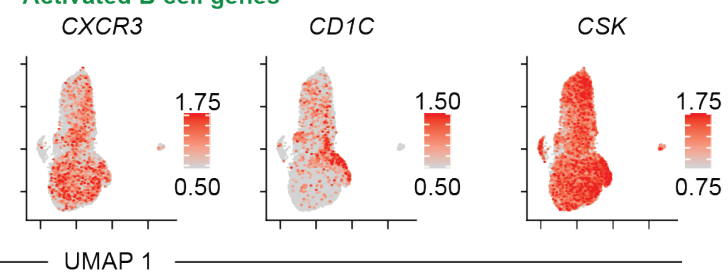

UMAP 1

Figure 2: High throughput single cell analysis of reveals the full diversity of circulating $B$ cell populations. Single B cells were sorted from 2 malaria exposed Kenyan individuals and 2 Australian individuals and gene expression was assessed using 10x chromium methodology A. Unsupervised clustering of circulating mature $\mathrm{IgD}^{-} \mathrm{B}$ cells pooled from all individuals visualized using UMAP. Each cell is represented by a point and colored by cluster. B. Phylogenetic tree based on the 'average cell' from each cluster showing relationships in gene expression patterns between clusters. C. Heatmap displaying the normalized enrichment scores of multiple GSEA comparing each cluster against previously published gene sets. D, E \& F. Expression of atBC (D), antigen presentation (E), MBC and $\mathrm{ABC}(\mathrm{F})$ genes projected onto UMAP plots. Color was scaled for each marker with highest and lowest $\log$-normalized expression level noted. Where the exact $\mathrm{p}$ value is not quoted $* \mathrm{p}<0.05,{ }^{* *} \mathrm{p}<0.01$, $* * * \mathrm{P}<0.001$. 
a

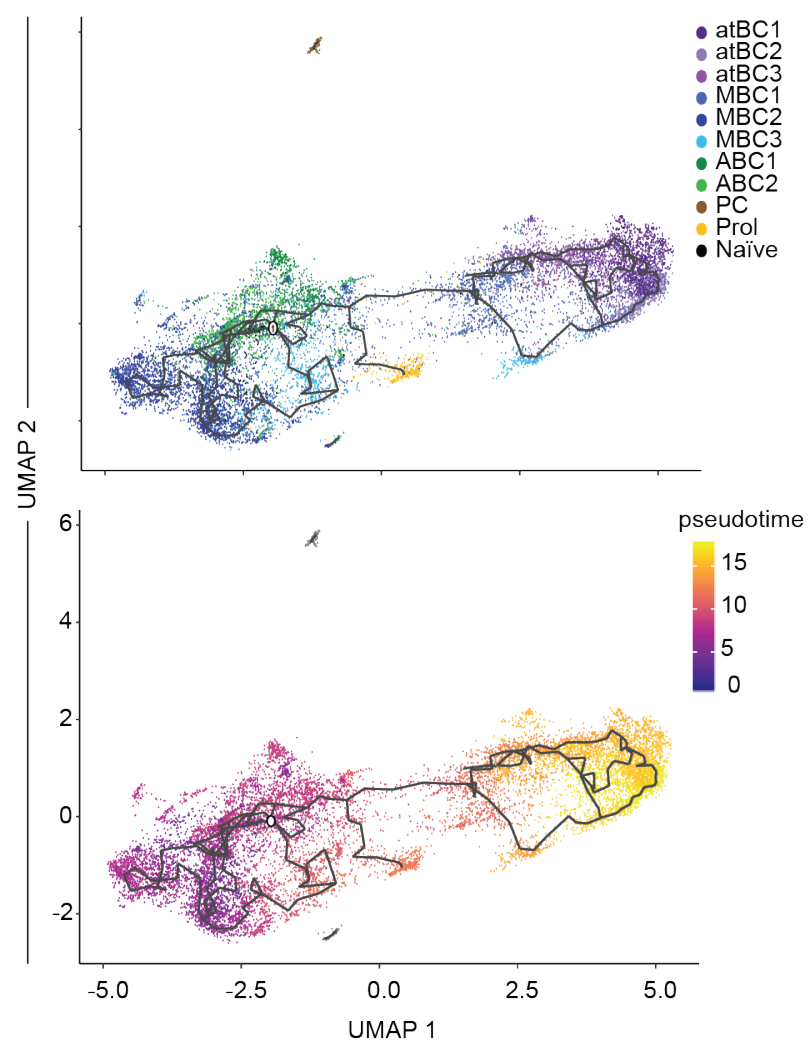

b

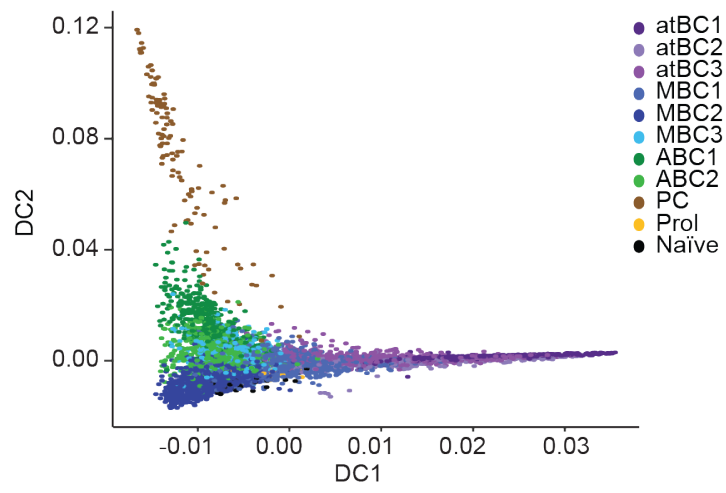

710 Figure 3: Pseudotime analysis and diffusion mapping reveal 2 distinct lineages of circulating $B$

711 cells. A. Pseudotime analysis of circulating B cells generated visualized using UMAP. Each point represents a cell and is colored by cluster or progression along pseudotime. B. Diffusion map showing

713 diffusion components (DC) 1 and 2, each cell is represented by a point and colored by cluster. 

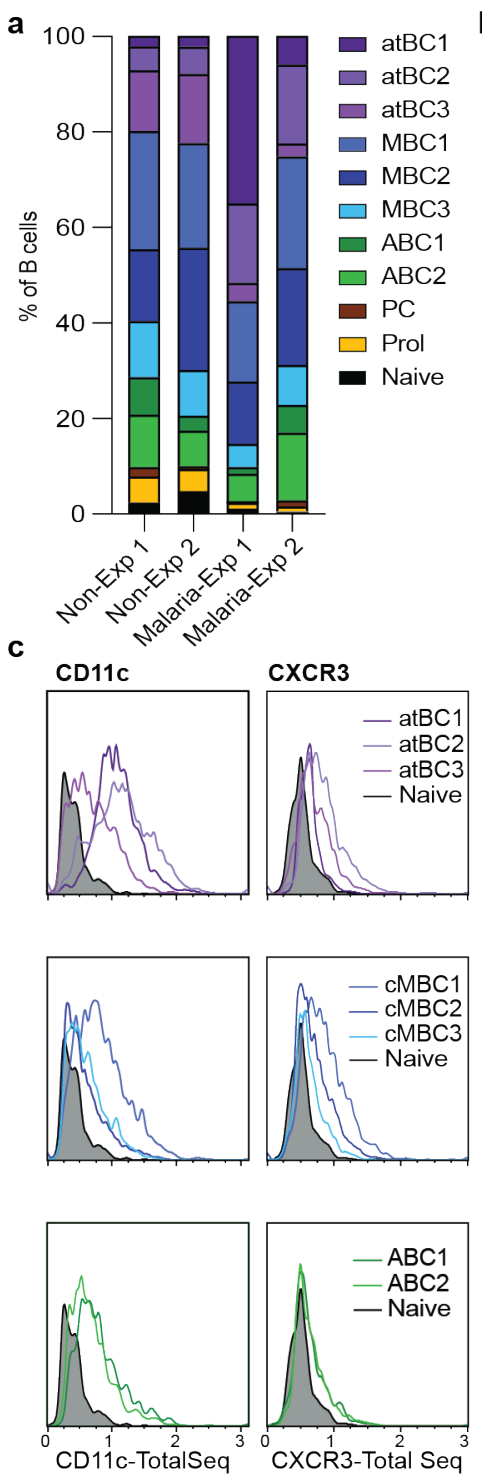

b

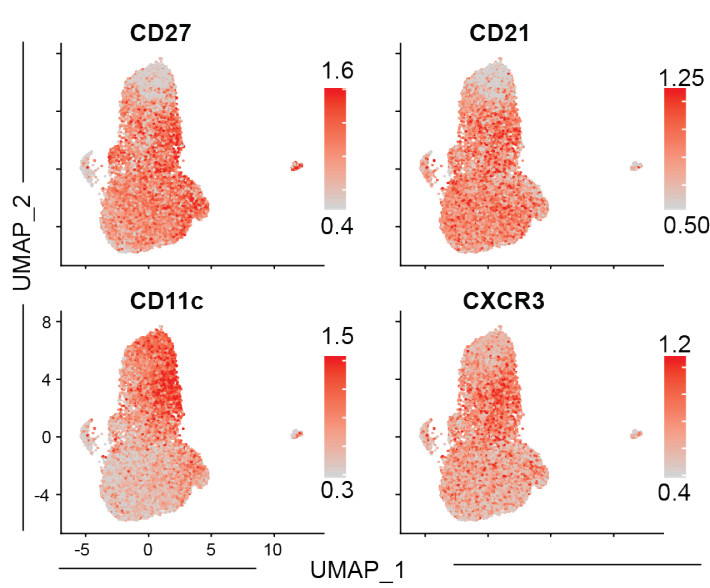

d

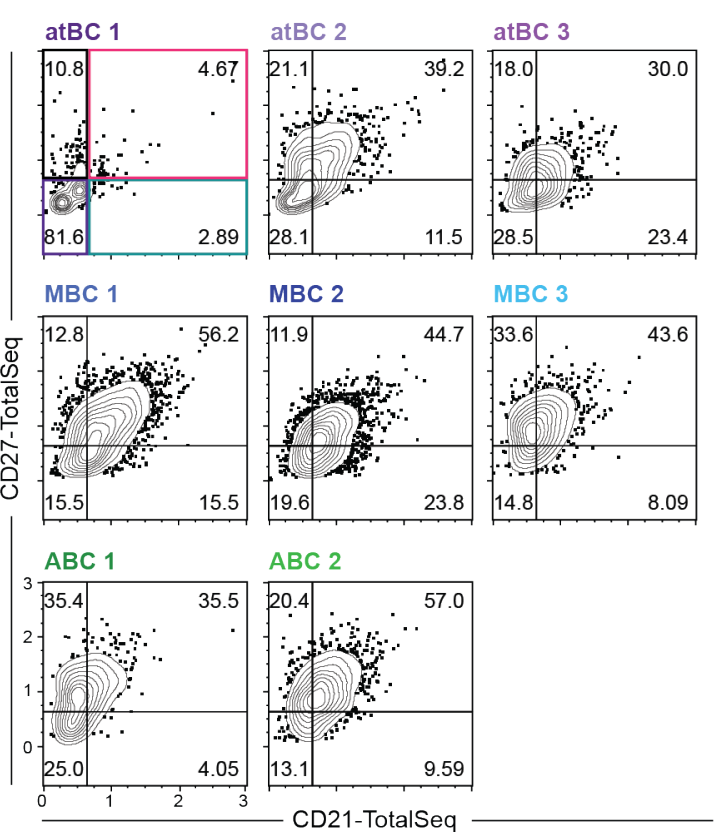

e

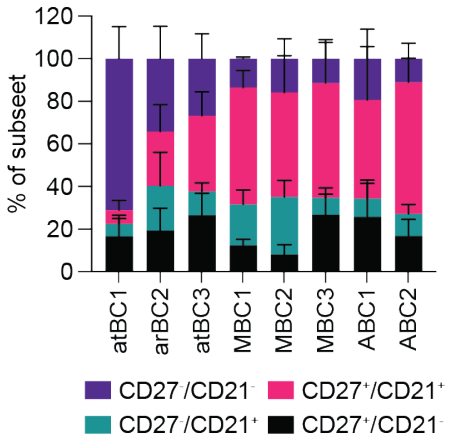

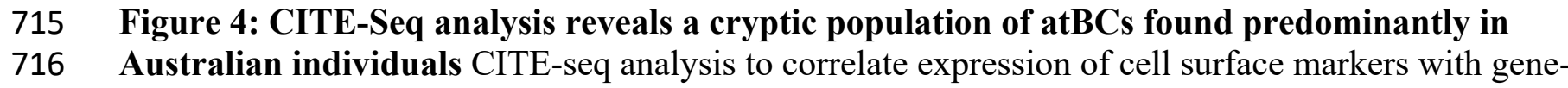
717 expression was performed on cells from the four donors described in figure 2 A. Percentage of cells 718 from each individual found in each cluster. B. Surface protein expression measured by CITE-seq 719 projected onto UMAP plots. Color was scaled for each marker with highest and lowest log-normalized expression level noted. C. Histogram plots showing the expression of CD11c and CXCR3 for the different atBC, $\mathrm{MBC}$ and $\mathrm{ABC}$ clusters, grey histogram represents expression on naïve $\mathrm{B}$ cells; data are concatenated from all individuals. D. Contour plots showing the expression of CD27 and CD21 as measured by CITE-seq; data are concatenated from all individuals E. Quantification of (D), data show the mean proportion per individual \pm s.d.. 
a

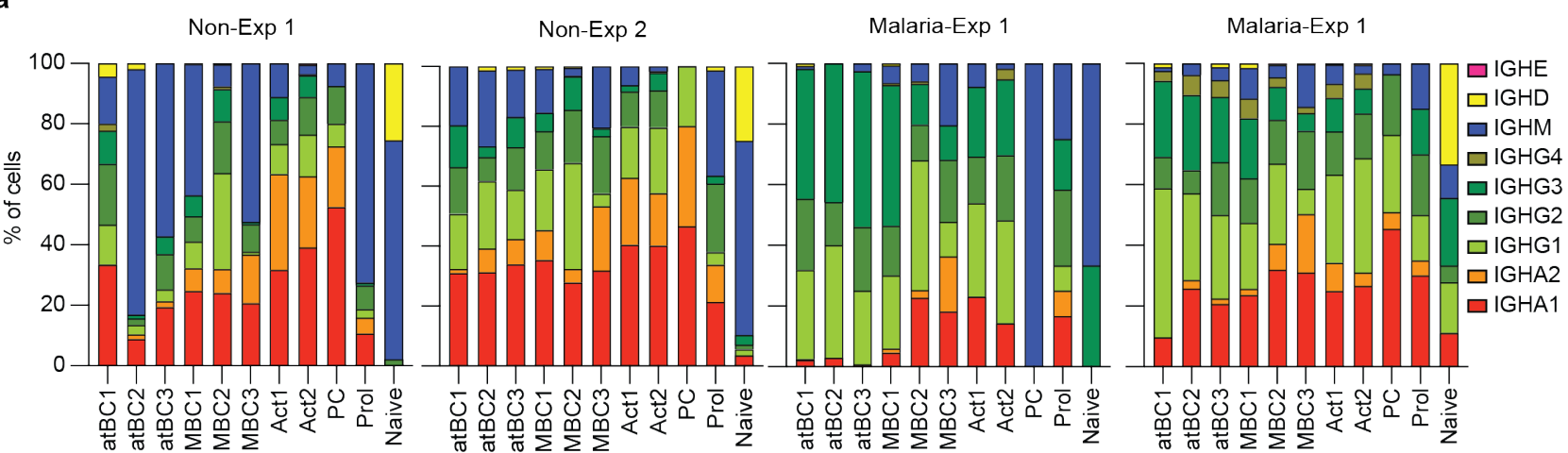

b

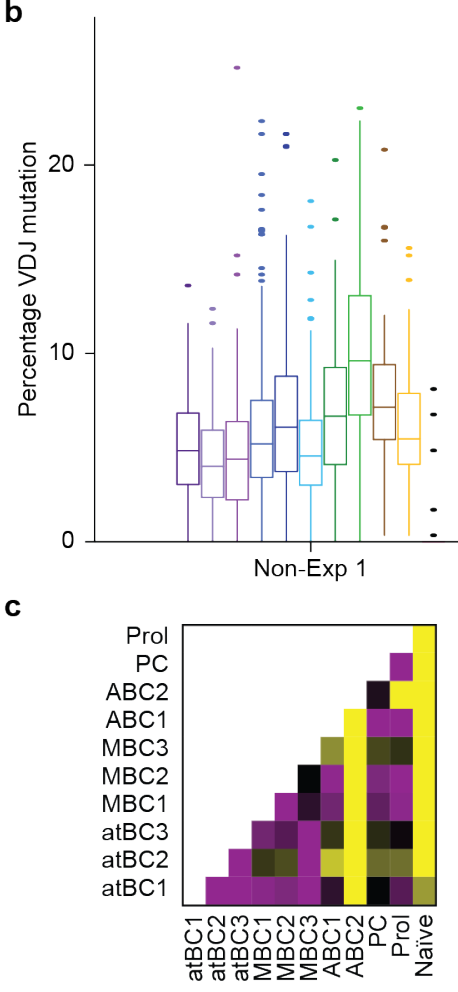

Non-Exp 1

d
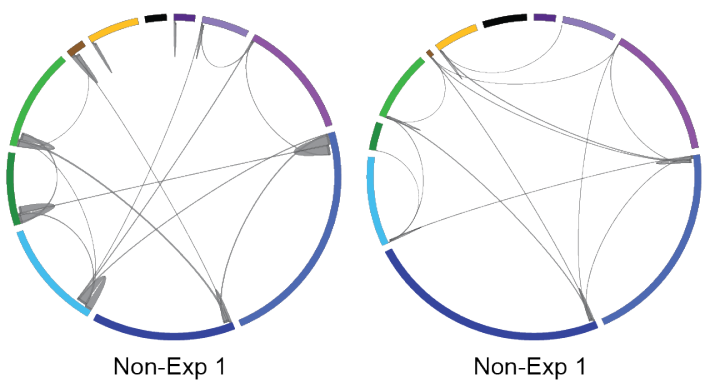

Non-Exp 1

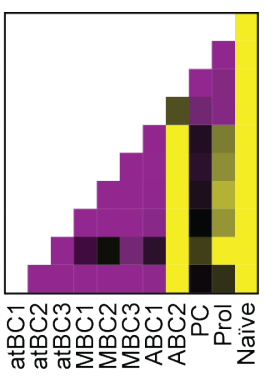

Non-Exp 1
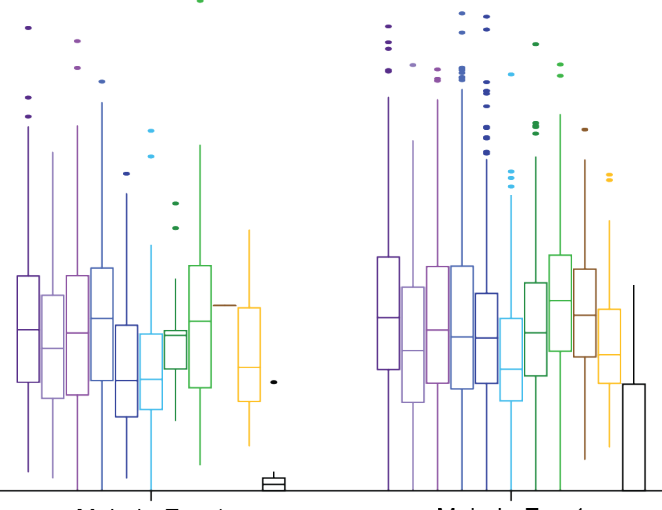

투 atBC1

追 atBC2

白 $\mathrm{atBC3}$

追 $\mathrm{MBC} 1$

白 $\mathrm{MBC2}$

타 $\mathrm{MBC} 3$

白 $A B C 1$

追 $\mathrm{ABC} 2$

白 $\mathrm{PC}$

Prol

官 Naive

Figure 5: Lack of association between BCR variable and constant regions with different $B$ cell subsets $V(D) J$ and constant region sequences for each cell from each donor described in figure 2 were mapped to the individual transcriptomes and relationships analyzed A. percentage of isotype usage for each cluster per individual. B. Percentage of mutations found in the heavy chain V(D)J region of cells for each cluster in each donor, mean \pm s.d. shown. C. Heatmaps displaying the pairwise p-values from 
bioRxiv preprint doi: https://doi.org/10.1101/2020.05.28.120808; this version posted May 28, 2020. The copyright holder for this preprint (which

was not certified by peer review) is the author/funder, who has granted bioRxiv a license to display the preprint in perpetuity. It is made available under aCC-BY-NC-ND 4.0 International license.

732 cell type and mutation frequency with subclass and individual also included in the model as fixed 733 factors. D. Circos plots showing clonal B cell populations per individual, the thickness of the lines 734 between or within clusters denotes the number of cells that belong to shared/expanded clones. 
a

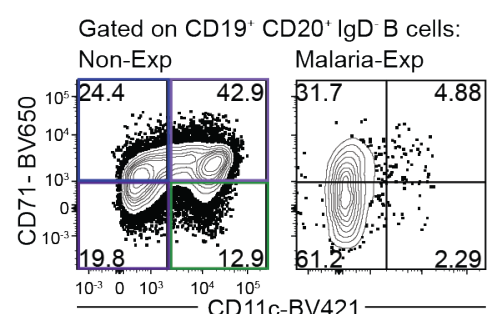

C
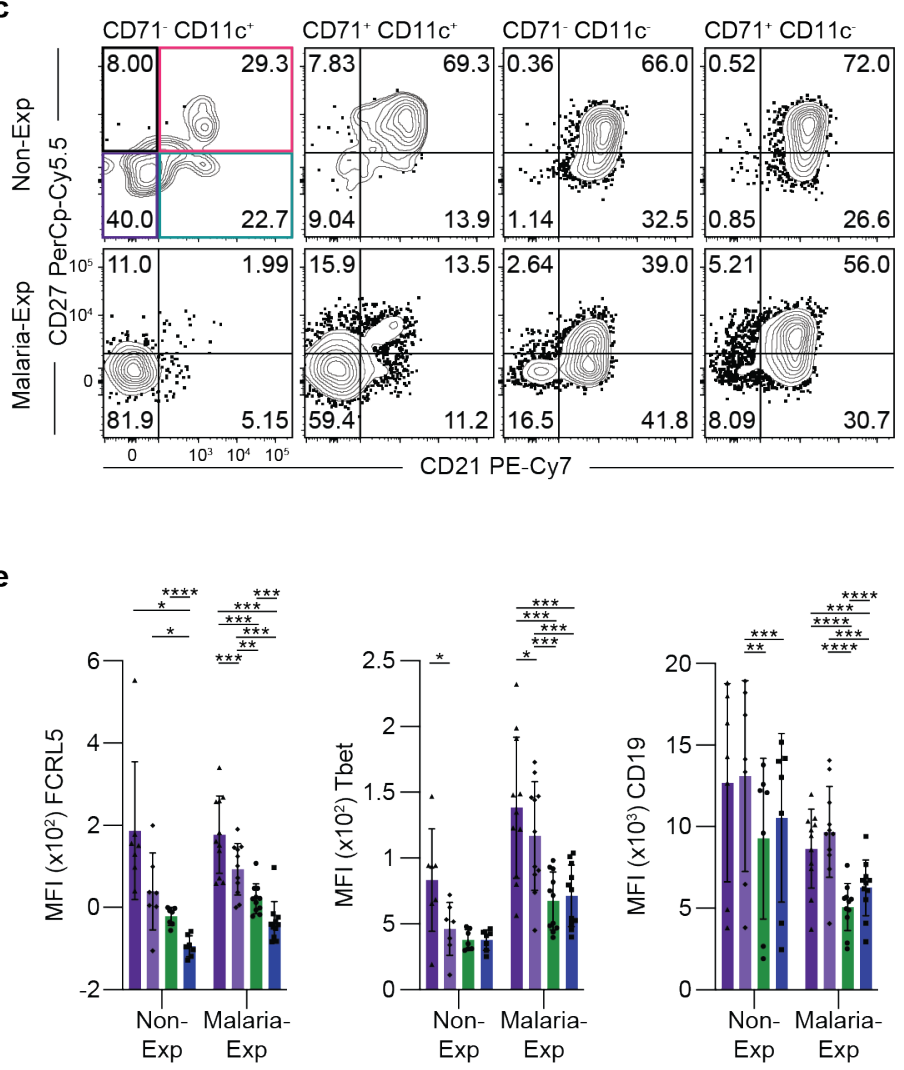

b

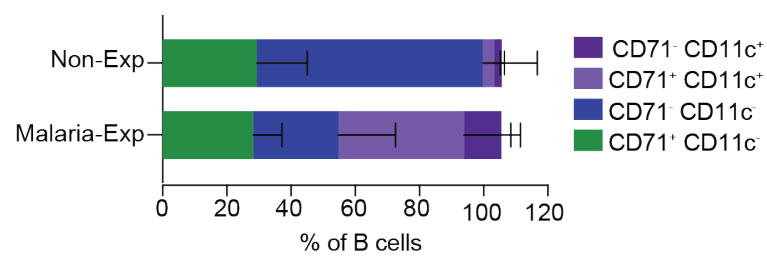

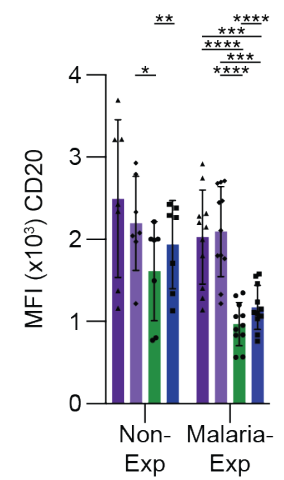

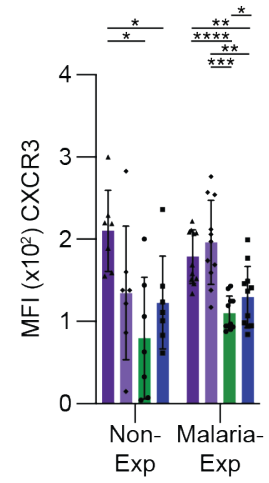

$\mathrm{CD} 71-\mathrm{CD} 11 \mathrm{c}$ $\mathrm{CD} 71^{+} \mathrm{CD} 11 \mathrm{c}^{+}$ CD71 CD11c $\mathrm{CD} 71^{+} \mathrm{CD} 11 \mathrm{c}$

Figure 6: CD11c and CD71 identify atBC, $\mathrm{ABCs}$ and $\mathrm{MBCs}$ via flow cytometry PBMCs from 7 Non-Exp and 11 Malaria-Exp donors were isolated and analyzed by flow cytometry for expression of markers associated with different B cell populations A. Flow cytometry plots from representative individuals showing the CD11c and CD71 expression on mature IgD-B cells. B. Quantification of (A) showing the percentage of cells found in each cell type by country, bars represent mean \pm s.d.. C. Representative flow cytometry plots showing the expression of CD27 and CD21 per cell type. D. Quantification of (C) showing the percentage of cells separated by expression of CD27 and CD21 found in each cell type, bars represent the mean proportion \pm s.d.. E. The expression of surfaces markers on each cell type, measured by MFI, analysis was done using 2-way ANOVA, bars represent mean \pm s.d.. 
a
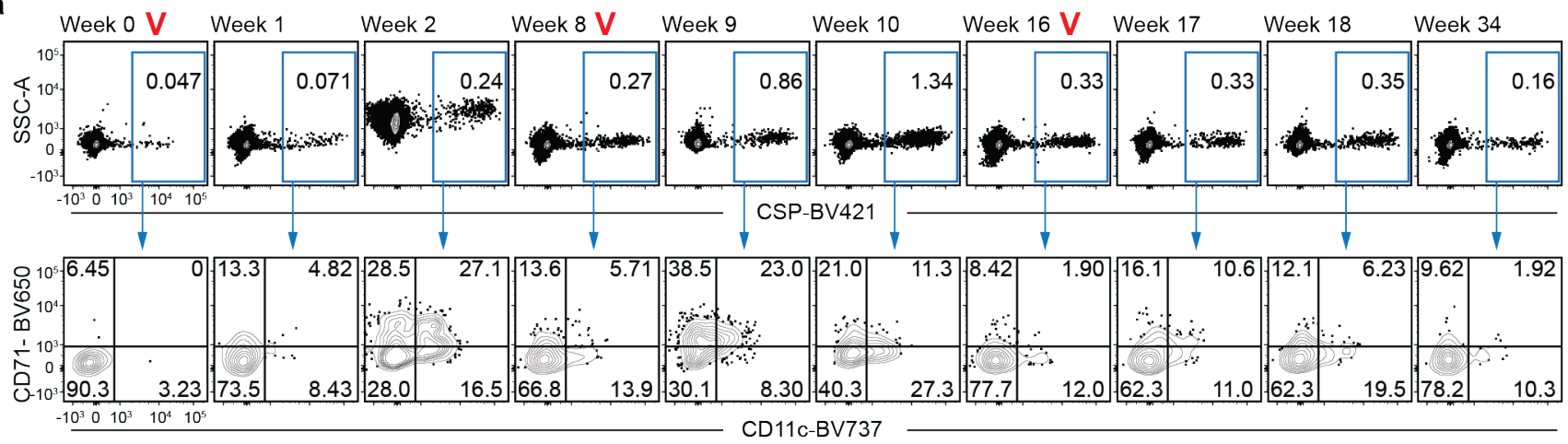

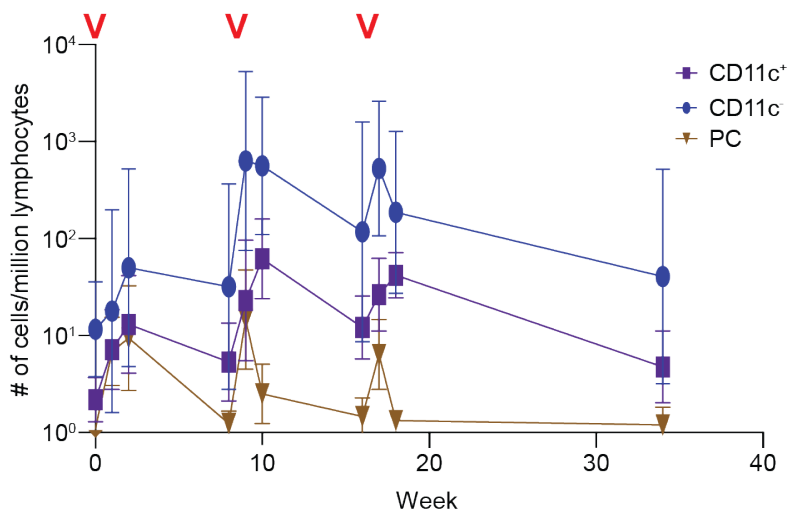

d

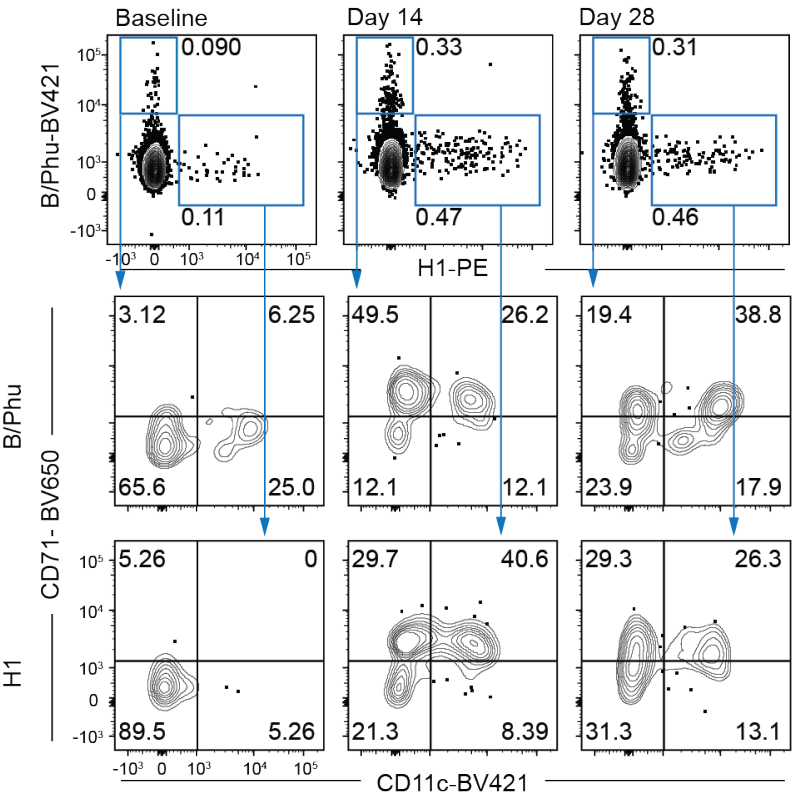

C

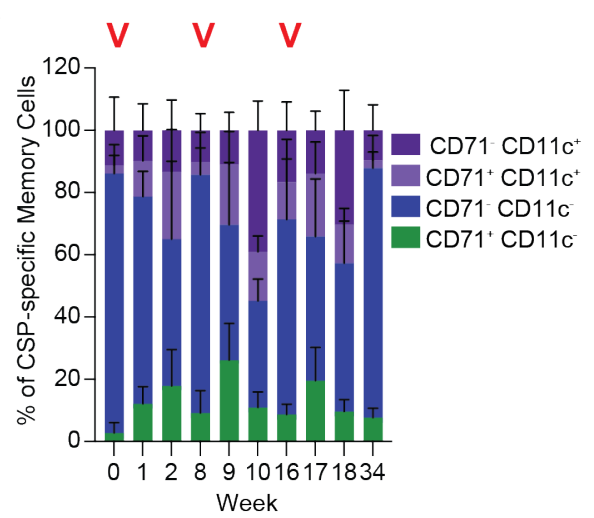

e
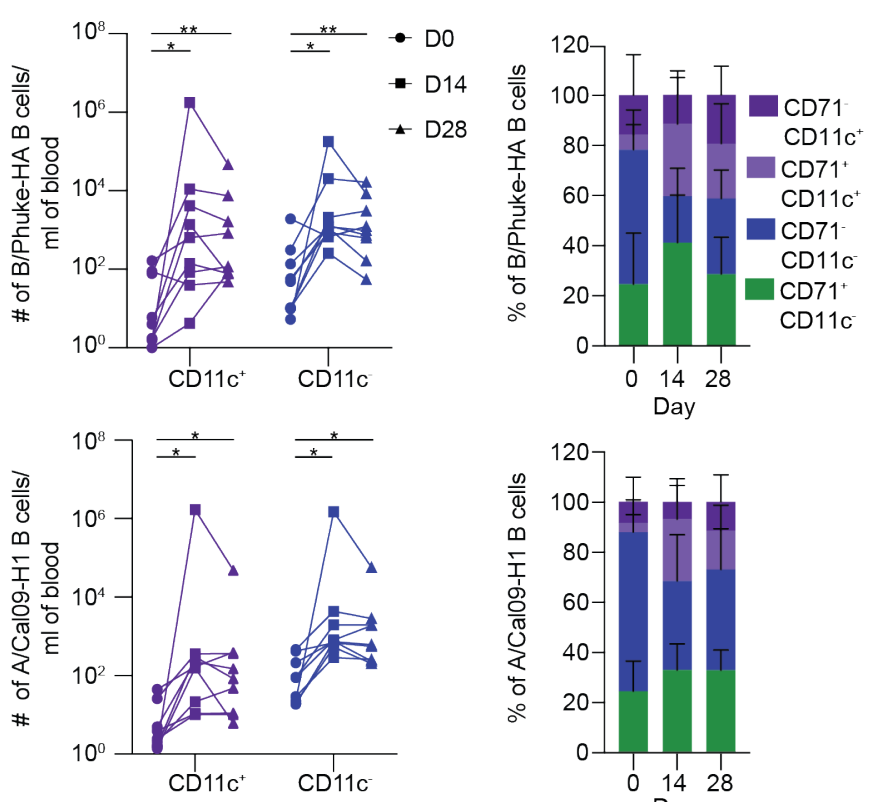

747 Figure 7: Antigen-specific atBCs arise during the primary response and can be effectively

748 recalled. A. 15 individuals were vaccinated with 3 doses of 9x105 PfSPZ at 8-week intervals, with 749 blood drawn at the indicated timepoints; panel shows representative flow cytometry plots from a single 750 individual of the gating of CSP-specific IgD- B cells and the CD71 and CD11c expression found in 751 these cells over time. Red "V"s indicate time points where booster immunizations were given. B.

752 Kinetics of the CSP-specific B cell response quantified by the number of cells per million lymphocytes. 753 C. The percentage of CSP-specific memory cells divided by CD11c and CD71 expression over time D. 
7549 individuals were vaccinated with inactivated influenza vaccine (IIV) with blood drawn at baseline 755 and 14 or 28 days later; panel shows representative flow cytometry plots showing the number of either $756 \mathrm{~B} /$ Phuket or H1-specific IgD- B cells and the CD71 and CD11c expression found in these cells over 757 time. E. Kinetics of the influenza-specific B cell response quantified by the number of cells per $\mathrm{mL}$ of 758 blood. F. The percentage of influenza-specific memory cells divided by CD11c and CD71 expression. 
References

Angerer, P., Haghverdi, L., Buttner, M., Theis, F.J., Marr, C., and Buettner, F. (2016). destiny:

Arroyo, E.N., and Pepper, M. (2020). B cells are sufficient to prime the dominant CD4+ Tfh response to Plasmodium infection. J Exp Med 217.

Avery, D.T., Ellyard, J.I., Mackay, F., Corcoran, L.M., Hodgkin, P.D., and Tangye, S.G. (2005). Increased expression of CD27 on activated human memory B cells correlates with their commitment to the plasma cell lineage (vol 174, pg 4034, 2005). Journal of Immunology 174, 5885-5885.

Aye, R., Sutton, H.J., Nduati, E.W., Kai, O., Mwacharo, J., Musyoki, J., Otieno, E., Wambua, J., Bejon, P., Cockburn, I.A., et al. (2020). Malaria exposure drives both cognate and bystander human B cells to adopt an atypical phenotype. Eur J Immunol.

Barnett, B.E., Staupe, R.P., Odorizzi, P.M., Palko, O., Tomov, V.T., Mahan, A.E., Gunn, B., Chen, D., Paley, M.A., Alter, G., et al. (2016). Cutting Edge: B Cell-Intrinsic T-bet Expression Is Required To Control Chronic Viral Infection. J Immunol 197, 1017-1022.

Baumjohann, D., Preite, S., Reboldi, A., Ronchi, F., Ansel, K.M., Lanzavecchia, A., and Sallusto, F. (2013). Persistent antigen and germinal center B cells sustain T follicular helper cell responses and phenotype. Immunity 38, 596-605.

Butler, A., Hoffman, P., Smibert, P., Papalexi, E., and Satija, R. (2018). Integrating single-cell transcriptomic data across different conditions, technologies, and species. Nat Biotechnol 36, 411-420.

Cockburn, I.A., Chen, Y.C., Overstreet, M.G., Lees, J.R., van Rooijen, N., Farber, D.L., and Zavala, F. (2010). Prolonged antigen presentation is required for optimal CD8+ T cell responses against malaria liver stage parasites. PLoS Pathog 6, e1000877.

Ehrhardt, G.R.A., Hsu, J.T., Gartland, L., Leu, C.M., Zhang, S.Y., Davis, R.S., and Cooper, M.D. (2005). Expression of the immunoregulatory molecule FcRH4 defines a distinctive tissue-based population of memory B cells. Journal of Experimental Medicine 202, 783-791.

Ellebedy, A.H., Jackson, K.J., Kissick, H.T., Nakaya, H.I., Davis, C.W., Roskin, K.M., McElroy, A.K., Oshansky, C.M., Elbein, R., Thomas, S., et al. (2016). Defining antigen-specific plasmablast and memory B cell subsets in human blood after viral infection or vaccination. Nat Immunol 17, 12261234.

Fecteau, J.F., Cote, G., and Neron, S. (2006). A new memory CD27-IgG+ B cell population in peripheral blood expressing VH genes with low frequency of somatic mutation. J Immunol 177, 3728 3736. 
803

804

805

806

807

808

809

810

811

812

813

814

815

816

817

818

819

820

821

822

823

824

825

826

827

828

829

830

831

832

833

834

835

836

837

838

839

840

841

842

843

844

845

846

847

848

849

850
Good, K.L., Avery, D.T., and Tangye, S.G. (2009). Resting Human Memory B Cells Are Intrinsically Programmed for Enhanced Survival and Responsiveness to Diverse Stimuli Compared to Naive B Cells. Journal of Immunology 182, 890-901.

Guehenneux, F., Duret, L., Callanan, M.B., Bouhas, R., Hayette, S., Berthet, C., Samarut, C., Rimokh, R., Birot, A.M., Wang, Q., et al. (1997). Cloning of the mouse BTG3 gene and definition of a new gene family (the BTG family) involved in the negative control of the cell cycle. Leukemia 11,370-375.

Horst, A., Hunzelmann, N., Arce, S., Herber, M., Manz, R.A., Radbruch, A., Nischt, R., Schmitz, J., and Assenmacher, M. (2002). Detection and characterization of plasma cells in peripheral blood: correlation of IgE+ plasma cell frequency with IgE serum titre. Clin Exp Immunol 130, 370-378.

Illingworth, J., Butler, N.S., Roetynck, S., Mwacharo, J., Pierce, S.K., Bejon, P., Crompton, P.D., Marsh, K., and Ndungu, F.M. (2013). Chronic exposure to Plasmodium falciparum is associated with phenotypic evidence of B and T cell exhaustion. J Immunol 190, 1038-1047.

Ishizuka, A.S., Lyke, K.E., DeZure, A., Berry, A.A., Richie, T.L., Mendoza, F.H., Enama, M.E., Gordon, I.J., Chang, L.J., Sarwar, U.N., et al. (2016). Protection against malaria at 1 year and immune correlates following PfSPZ vaccination. Nat Med 22, 614-623.

Isnardi, I., Ng, Y.S., Menard, L., Meyers, G., Saadoun, D., Srdanovic, I., Samuels, J., Berman, J., Buckner, J.H., Cunningham-Rundles, C., et al. (2010). Complement receptor 2/CD21(-) human naive B cells contain mostly autoreactive unresponsive clones. Blood 115, 5026-5036.

Jenks, S.A., Cashman, K.S., Zumaquero, E., Marigorta, U.M., Patel, A.V., Wang, X., Tomar, D., Woodruff, M.C., Simon, Z., Bugrovsky, R., et al. (2018). Distinct Effector B Cells Induced by Unregulated Toll-like Receptor 7 Contribute to Pathogenic Responses in Systemic Lupus Erythematosus. Immunity 49, 725-739 e726.

Kim, C.C., Baccarella, A.M., Bayat, A., Pepper, M., and Fontana, M.F. (2019). FCRL5(+) Memory B Cells Exhibit Robust Recall Responses. Cell Rep 27, 1446-1460 e1444.

Klein, U., Casola, S., Cattoretti, G., Shen, Q., Lia, M., Mo, T., Ludwig, T., Rajewsky, K., and DallaFavera, R. (2006). Transcription factor IRF4 controls plasma cell differentiation and class-switch recombination. Nat Immunol 7, 773-782.

Klein, U., Kuppers, R., and Rajewsky, K. (1997). Evidence for a large compartment of IgM-expressing memory B cells in humans. Blood 89, 1288-1298.

Knox, J.J., Buggert, M., Kardava, L., Seaton, K.E., Eller, M.A., Canaday, D.H., Robb, M.L., Ostrowski, M.A., Deeks, S.G., Slifka, M.K., et al. (2017). T-bet+ B cells are induced by human viral infections and dominate the HIV gp140 response. JCI Insight 2.

Koutsakos, M., Wheatley, A.K., Loh, L., Clemens, E.B., Sant, S., Nussing, S., Fox, A., Chung, A.W., Laurie, K.L., Hurt, A.C., et al. (2018). Circulating T-FH cells, serological memory, and tissue compartmentalization shape human influenza-specific B cell immunity. Science Translational Medicine 10 . 
851

852

853

854

855

856

857

858

859

860

861

862

863

864

865

866

867

868

869

870

871

872

873

874

875

876

877

878

879

880

881

882

883

884

885

886

887

888

889

890

891

892

893

894

895

896

897

898

Krishnamurty, A.T., Thouvenel, C.D., Portugal, S., Keitany, G.J., Kim, K.S., Holder, A., Crompton, P.D., Rawlings, D.J., and Pepper, M. (2016). Somatically Hypermutated Plasmodium-Specific IgM(+) Memory B Cells Are Rapid, Plastic, Early Responders upon Malaria Rechallenge. Immunity 45, 402414.

Lau, D., Lan, L.Y., Andrews, S.F., Henry, C., Rojas, K.T., Neu, K.E., Huang, M., Huang, Y., DeKosky, B., Palm, A.E., et al. (2017). Low CD21 expression defines a population of recent germinal center graduates primed for plasma cell differentiation. Sci Immunol 2.

Lyke, K.E., Ishizuka, A.S., Berry, A.A., Chakravarty, S., DeZure, A., Enama, M.E., James, E.R., Billingsley, P.F., Gunasekera, A., Manoj, A., et al. (2017). Attenuated PfSPZ Vaccine induces straintranscending $\mathrm{T}$ cells and durable protection against heterologous controlled human malaria infection. Proc Natl Acad Sci U S A 114, 2711-2716.

Moir, S., Ho, J., Malaspina, A., Wang, W., DiPoto, A.C., O'Shea, M.A., Roby, G., Kottilil, S., Arthos, J., Proschan, M.A., et al. (2008). Evidence for HIV-associated B cell exhaustion in a dysfunctional memory B cell compartment in HIV-infected viremic individuals. J Exp Med 205, 1797-1805.

Muellenbeck, M.F., Ueberheide, B., Amulic, B., Epp, A., Fenyo, D., Busse, C.E., Esen, M., Theisen, M., Mordmuller, B., and Wardemann, H. (2013). Atypical and classical memory B cells produce Plasmodium falciparum neutralizing antibodies. J Exp Med 210, 389-399.

Murugan, R., Buchauer, L., Triller, G., Kreschel, C., Costa, G., Pidelaserra Marti, G., Imkeller, K., Busse, C.E., Chakravarty, S., Sim, B.K.L., et al. (2018). Clonal selection drives protective memory B cell responses in controlled human malaria infection. Sci Immunol 3.

Muto, A., Hoshino, H., Madisen, L., Yanai, N., Obinata, M., Karasuyama, H., Hayashi, M., Nakauchi, H., Yamamoto, M., Groudine, M., et al. (1998). Identification of Bach2 as a B-cell-specific partner for small Maf proteins that negatively regulate the immunoglobulin heavy chain gene 3 ' enhancer. Embo Journal 17, 5734-5743.

Obeng-Adjei, N., Portugal, S., Holla, P., Li, S., Sohn, H., Ambegaonkar, A., Skinner, J., Bowyer, G., Doumbo, O.K., Traore, B., et al. (2017). Malaria-induced interferon-gamma drives the expansion of Tbethi atypical memory B cells. PLoS Pathog 13, e1006576.

Pape, K.A., Taylor, J.J., Maul, R.W., Gearhart, P.J., and Jenkins, M.K. (2011). Different B cell populations mediate early and late memory during an endogenous immune response. Science 331, 1203-1207.

Perez-Mazliah, D., Gardner, P.J., Schweighoffer, E., McLaughlin, S., Hosking, C., Tumwine, I., Davis, R.S., Potocnik, A.J., Tybulewicz, V.L., and Langhorne, J. (2018). Plasmodium-specific atypical memory B cells are short-lived activated B cells. Elife 7.

Picelli, S., Faridani, O.R., Bjorklund, A.K., Winberg, G., Sagasser, S., and Sandberg, R. (2014). Fulllength RNA-seq from single cells using Smart-seq2. Nat Protoc 9, 171-181.

Plotkin, S.A. (2010). Correlates of protection induced by vaccination. Clin Vaccine Immunol 17, 10551065. 
Portugal, S., Tipton, C.M., Sohn, H., Kone, Y., Wang, J., Li, S., Skinner, J., Virtaneva, K., Sturdevant, D.E., Porcella, S.F., et al. (2015). Malaria-associated atypical memory B cells exhibit markedly reduced $\mathrm{B}$ cell receptor signaling and effector function. Elife 4.

Reimold, A.M., Iwakoshi, N.N., Manis, J., Vallabhajosyula, P., Szomolanyi-Tsuda, E., Gravallese, E.M., Friend, D., Grusby, M.J., Alt, F., and Glimcher, L.H. (2001). Plasma cell differentiation requires the transcription factor XBP-1. Nature 412, 300-307.

Rivera-Correa, J., Mackroth, M.S., Jacobs, T., Schulze Zur Wiesch, J., Rolling, T., and Rodriguez, A. (2019). Atypical memory B-cells are associated with Plasmodium falciparum anemia through antiphosphatidylserine antibodies. Elife 8.

Rizzetto, S., Koppstein, D.N.P., Samir, J., Singh, M., Reed, J.H., Cai, C.H., Lloyd, A.R., Eltahla, A.A., Goodnow, C.C., and Luciani, F. (2018). B-cell receptor reconstruction from single-cell RNA-seq with VDJPuzzle. Bioinformatics 34, 2846-2847.

Rubtsov, A.V., Rubtsova, K., Kappler, J.W., Jacobelli, J., Friedman, R.S., and Marrack, P. (2015). CD11c-Expressing B Cells Are Located at the T Cell/B Cell Border in Spleen and Are Potent APCs. J Immunol 195, 71-79.

Rubtsova, K., Rubtsov, A.V., van Dyk, L.F., Kappler, J.W., and Marrack, P. (2013). T-box transcription factor T-bet, a key player in a unique type of B-cell activation essential for effective viral clearance. Proc Natl Acad Sci U S A 110, E3216-3224.

Shaffer, A.L., Lin, K.I., Kuo, T.C., Yu, X., Hurt, E.M., Rosenwald, A., Giltnane, J.M., Yang, L.M., Zhao, H., Calame, K., et al. (2002). Blimp-1 orchestrates plasma cell differentiation by extinguishing the mature B cell gene expression program. Immunity 17, 51-62.

Stoeckius, M., Hafemeister, C., Stephenson, W., Houck-Loomis, B., Chattopadhyay, P.K., Swerdlow, H., Satija, R., and Smibert, P. (2017). Simultaneous epitope and transcriptome measurement in single cells. Nat Methods 14, 865-868.

Sullivan, R.T., Kim, C.C., Fontana, M.F., Feeney, M.E., Jagannathan, P., Boyle, M.J., Drakeley, C.J., Ssewanyana, I., Nankya, F., Mayanja-Kizza, H., et al. (2015). FCRL5 Delineates Functionally Impaired Memory B Cells Associated with Plasmodium falciparum Exposure. PLoS Pathog 11, e1004894.

Tan, J., Sack, B.K., Oyen, D., Zenklusen, I., Piccoli, L., Barbieri, S., Foglierini, M., Fregni, C.S., Marcandalli, J., Jongo, S., et al. (2018). A public antibody lineage that potently inhibits malaria infection through dual binding to the circumsporozoite protein. Nat Med 24, 401-407.

Tangye, S.G., Avery, D.T., Deenick, E.K., and Hodgkin, P.D. (2003). Intrinsic differences in the proliferation of naive and memory human B cells as a mechanism for enhanced secondary immune responses. Journal of Immunology 170, 686-694.

Tangye, S.G., Liu, Y.J., Aversa, G., Phillips, J.H., and de Vries, J.E. (1998). Identification of functional human splenic memory B cells by expression of CD148 and CD27. J Exp Med 188, 1691-1703. 
946 Trapnell, C., Cacchiarelli, D., Grimsby, J., Pokharel, P., Li, S., Morse, M., Lennon, N.J., Livak, K.J., 947 Mikkelsen, T.S., and Rinn, J.L. (2014). The dynamics and regulators of cell fate decisions are revealed 948 by pseudotemporal ordering of single cells. Nat Biotechnol 32, 381-386.

950 Tsukumo, S., Unno, M., Muto, A., Takeuchi, A., Kometani, K., Kurosaki, T., Igarashi, K., and Saito, T. 951 (2013). Bach2 maintains T cells in a naive state by suppressing effector memory-related genes. Proc 952 Natl Acad Sci U S A 110, 10735-10740.

954 Wei, C., Anolik, J., Cappione, A., Zheng, B., Pugh-Bernard, A., Brooks, J., Lee, E.H., Milner, E.C., 955 and Sanz, I. (2007). A new population of cells lacking expression of CD27 represents a notable 956 component of the B cell memory compartment in systemic lupus erythematosus. J Immunol 178, 66249576633.

959 Weiss, G.E., Crompton, P.D., Li, S., Walsh, L.A., Moir, S., Traore, B., Kayentao, K., Ongoiba, A., 960 Doumbo, O.K., and Pierce, S.K. (2009). Atypical memory B cells are greatly expanded in individuals 961 living in a malaria-endemic area. J Immunol 183, 2176-2182. 
bioRxiv preprint doi: https://doi.org/10.1101/2020.05.28.120808; this version posted May 28, 2020. The copyright holder for this preprint (which

was not certified by peer review) is the author/funder, who has granted bioRxiv a license to display the preprint in perpetuity. It is made available under aCC-BY-NC-ND 4.0 International license. 